\title{
A Highly Regioselective Amination of 6-Aryl-2,4-Dichloropyrimidine
}

\author{
Zhi-Hui Peng*, Michel Journet, and Guy Humphrey \\ Department of Process Research, Merck \& Co., Inc., P. O. Box 2000, Rahway, NJ 07065
}

\section{Supporting Information}

\section{Contents:}

I. Synthesis of 6-aryl-2,4-dichloropyrimidines via Suzuki reactions..............................

II. General procedure for the nucleophilic aromatic substitution of 6-aryl-2,4-dichloropyrimidines with

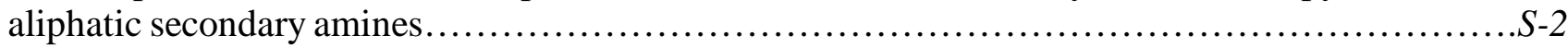

III. General procedure for the nucleophilic aromatic substitution of 6-aryl-2,4-dichloropyrimidines with

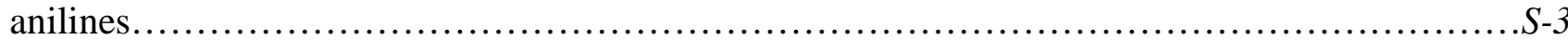

IV. General procedure for the Pd-catalyzed amination of 6-aryl-2,4-dichloropyrimidines with aliphatic

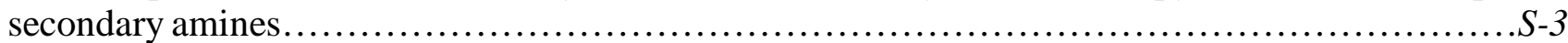

V. General procedure for the nucleophilic aromatic substitution of 6-aryl-2,4-dichloropyrimidines with

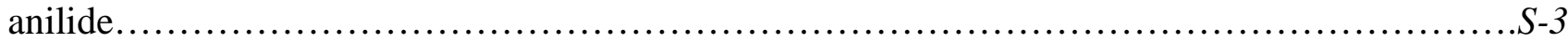

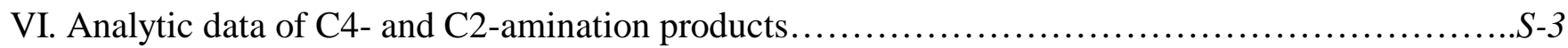

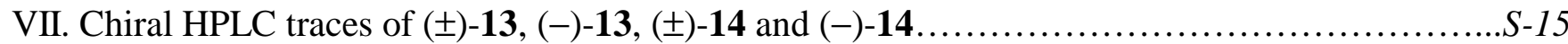

VIII. X-ray crystallographic determination information for compound $\mathbf{6} \ldots \ldots \ldots \ldots \ldots \ldots \ldots \ldots \ldots . . .17$

General: All reactions were run under an atmosphere of nitrogen using anhydrous solvents purchased from Aldrich. All reagents were purchased from Aldrich and used as received. All NMR spectra were recorded on a Bruker $400 \mathrm{MHz}$ instrument in $\mathrm{CDCl}_{3}$. HPLC analyses were carried out on a Hewlett Packard 1100 system. Enantiomeric excesses of $\mathbf{1 3}$ and $\mathbf{1 4}$ were determined by HPLC analyses using a Chiralpak AD-H column (250 $\times 4.6 \mathrm{~mm}$ ), and the enantiomerically enriched material was compared with racemic material. Melting points were determined on a Buchi B-545 apparatus and are uncorrected. Elemental analyses were preformed by Quantitative Technologies Inc., Whitehouse Station, NJ.

\section{Synthesis of 6-aryl-2,4-dichloropyrimidines via Suzuki reactions:}<smiles>Fc1ccc(-c2cc(Cl)nc(Cl)n2)cc1</smiles>

3<smiles>PP(Cl)(Cl)(c1ccccc1)c1cc(Cl)nc(Cl)n1</smiles>

6

2,4-Dichloro-6-(4-fluorophenyl)pyrimidine (3) and chloro-(2,6-dichloropyrimidin-4-yl)-bis-(triphenylphosphoranyl)palladium (6). To a solution of of 2,4,6-trichloropyrimidine $1(27.7 \mathrm{~g}, 150 \mathrm{mmol})$ in $100 \mathrm{~mL}$ of THF was added 4-fluorophenylboronic acid 2 (14.0 g, $100 \mathrm{mmol}), \mathrm{Pd}(\mathrm{OAc})_{2}(0.449 \mathrm{~g}, 2.00 \mathrm{mmol}), \mathrm{PPh}_{3}(1.05$ g, $4.00 \mathrm{mmol})$, followed by $\mathrm{Na}_{2} \mathrm{CO}_{3}(1 \mathrm{M}$ solution, $200 \mathrm{~mL}, 200 \mathrm{mmol})$. The reaction was heated to $60{ }^{\circ} \mathrm{C}$ for 3 $\mathrm{h}$ and was completed as indicated by the disappearance of the boronic acid by HPLC. The mixture was cooled 
and $100 \mathrm{~mL}$ of MTBE was added. The layers were separated, and the organic layer was washed with $100 \mathrm{~mL}$ of brine and concentrated in vacuo. The residue was purified by flash chromatography (5:95 to 20:80 EtOAc/hexanes) to yield 3 (21.8 g, 90\% yield) as a white solid and the palladium complex $\mathbf{6}$ (1.02 g) as a pale yellow solid. Recrystallization of $\mathbf{6}$ from EtOAc provided a single crystal suitable for X-ray analysis. 3: $\mathrm{mp}$ 143-145 ${ }^{\circ} \mathrm{C} ;{ }^{1} \mathrm{H}$ NMR $\left(\mathrm{CDCl}_{3}, 400 \mathrm{MHz}\right) \delta 8.07(\mathrm{~m}, 2 \mathrm{H}), 7.61(\mathrm{~s}, 1 \mathrm{H}), 7.19(\mathrm{~m}, 2 \mathrm{H}) ;{ }^{13} \mathrm{C} \mathrm{NMR}\left(\mathrm{CDCl}_{3}, 100\right.$ $\mathrm{MHz}) \delta 166.9,165.4(\mathrm{~d}, J=254.6 \mathrm{~Hz}), 163.0,160.9,130.2(\mathrm{~d}, J=3.2 \mathrm{~Hz}), 129.9(\mathrm{~d}, J=9.0 \mathrm{~Hz}), 116.4(\mathrm{~d}, J=$ 21.9 Hz), 114.9; Anal. Calcd for $\mathrm{C}_{10} \mathrm{H}_{5} \mathrm{Cl}_{2} \mathrm{FN}_{2}$ : C, 49.41; H, 2.07; N, 11.53. Found: C, 49.12; H, 1.97; N, 11.36. 6: $\mathrm{mp} 233-235{ }^{\circ} \mathrm{C} ;{ }^{1} \mathrm{H}$ NMR $\left(\mathrm{CDCl}_{3}, 400 \mathrm{MHz}\right) \delta 7.70(\mathrm{~m}, 12 \mathrm{H}), 7.45-7.27(\mathrm{~m}, 18 \mathrm{H}), 6.65(\mathrm{~s}, 1 \mathrm{H}) ;{ }^{13} \mathrm{C} \mathrm{NMR}$ $\left(\mathrm{CDCl}_{3}, 100 \mathrm{MHz}\right) \delta 207.2,155.2,154.9,134.6,134.5,134.46,134.4,134.3,130.6,130.3,130.2,130.0,129.7$, 129.5, 129.3, 129.2, 128.3, 128.25, 128.2, 128.1, 128.0, 127.9; Anal. Calcd for $\mathrm{C}_{40} \mathrm{H}_{31} \mathrm{Cl}_{3} \mathrm{~N}_{2} \mathrm{P}_{2} \mathrm{Pd}: \mathrm{C}, 58.99 ; \mathrm{H}$, 3.84 ; N, 3.44; Found: C, 58.82; H, 3.72; N, 3.45.<smiles>Clc1cc(-c2ccccc2)nc(Cl)n1</smiles>

7

2,4-Dichloro-6-phenylpyrimidine (7). Using the same procedure given for 3 with 1 (11.1 g, 60.0 mmol), phenylboronic acid $(6.10 \mathrm{~g}, 50.0 \mathrm{mmol}), \mathrm{Pd}(\mathrm{OAc})_{2}(0.225 \mathrm{~g}, 1.00 \mathrm{mmol}), \mathrm{PPh}_{3}(0.525 \mathrm{~g}, 2.00 \mathrm{mmol})$, and $\mathrm{Na}_{2} \mathrm{CO}_{3}$ (1 M solution, $100 \mathrm{~mL}, 100 \mathrm{mmol}$ ) afforded 7, after purification by flash chromatography (5:95 EtOAc/hexanes), as a white solid (10.0 g, 89\%): mp 92-93 ${ }^{\circ} \mathrm{C}$ (lit: $\left.{ }^{1} \mathrm{mp} 85-86{ }^{\circ} \mathrm{C}\right) ;{ }^{1} \mathrm{H} \mathrm{NMR}\left(\mathrm{CDCl}_{3}, 400 \mathrm{MHz}\right)$ $\delta 8.08(\mathrm{~m}, 2 \mathrm{H}), 7.69(\mathrm{~s}, 1 \mathrm{H}), 7.55(\mathrm{~m}, 3 \mathrm{H}) ;{ }^{13} \mathrm{C} \mathrm{NMR}\left(\mathrm{CDCl}_{3}, 100 \mathrm{MHz}\right) \delta 168.1,162.8,160.9,134.0,132.4$, $129.1,127.5,115.2$.<smiles>COc1ccc(-c2cc(Cl)nc(Cl)n2)cc1</smiles>

10

2,4-Dichloro-6-(4-methoxyphenyl)pyrimidine (10). Using the same procedure given for 3 with 1 (11.1 g, 60.0 mmol), 4-methoxyphenylboronic acid (7.60 g, $50.0 \mathrm{mmol}), \mathrm{Pd}(\mathrm{OAc})_{2}(0.225 \mathrm{~g}, 1.00 \mathrm{mmol}), \mathrm{PPh}_{3}(0.525 \mathrm{~g}, 2.00$ mmol), and $\mathrm{Na}_{2} \mathrm{CO}_{3}$ (1 M solution, $100 \mathrm{~mL}, 100 \mathrm{mmol}$ ) afforded 10, after purification by flash chromatography (5:95 EtOAc/hexanes), as a white solid (11.5 g, 90\%): $\mathrm{mp} 104-105{ }^{\circ} \mathrm{C} ;{ }^{1} \mathrm{H} \mathrm{NMR}\left(\mathrm{CDCl}_{3}, 400 \mathrm{MHz}\right) \delta 8.01(\mathrm{~d}, J$ $=8.9 \mathrm{~Hz}, 2 \mathrm{H}), 7.54(\mathrm{~s}, 1 \mathrm{H}), 6.98(\mathrm{~d}, J=8.9 \mathrm{~Hz}, 2 \mathrm{H}), 3.88(\mathrm{~s}, 3 \mathrm{H}) ;{ }^{13} \mathrm{C} \mathrm{NMR}\left(\mathrm{CDCl}_{3}, 100 \mathrm{MHz}\right) \delta 167.5,163.2$, 162.4, 160.7, 129.3, 126.3, 114.5, 114.0, 55.5; Anal. Calcd for $\mathrm{C}_{11} \mathrm{H}_{8} \mathrm{Cl}_{2} \mathrm{~N}_{2} \mathrm{O}$ : C, 51.79; H, 3.16; N, 10.98 . Found: C, 51.86; H, 3.05; N, 10.82 .

II. General procedure for the nucleophilic aromatic substitution of 6-aryl-2,4-dichloropyrimidines with aliphatic secondary amines: To a solution of 6-aryl-2,4-dichloropyrimidine (1.00 mmol) in $5 \mathrm{~mL}$ of DMAc was added amine $(1.00 \mathrm{mmol})$ and $\mathrm{K}_{2} \mathrm{CO}_{3}(2.00 \mathrm{mmol})$ at room temperature. The reation was monitored by TLC or HPLC. After the reaction was completed, the reaction mixture was diluted with $10 \mathrm{~mL}$ of water and was extracted with $3 \times 10 \mathrm{~mL}$ of EtOAc. The combined organic layers were washed with brine, dried $\left(\mathrm{Na}_{2} \mathrm{SO}_{4}\right)$ and 
concentrated in vacuo. Purification by flash chromatography (EtOAc/hexanes) provided both C4-substituted and C2-substituted products.

III. General procedure for the nucleophilic aromatic substitution of 6-aryl-2,4-dichloropyrimidines with anilines: To a solution of 6-aryl-2,4-dichloropyrimidine $(1.00 \mathrm{mmol})$ in $10 \mathrm{~mL}$ of 1-butanol was added aniline $(2.00 \mathrm{mmol})$ and $i-\mathrm{Pr}_{2} \mathrm{NEt}(2.00 \mathrm{mmol})$. The mixture was heated to reflux. After the reaction was completed as indicated by HPLC or TLC, the reaction mixture was diluted with $10 \mathrm{~mL}$ of saturated aqueous $\mathrm{NaHCO}_{3}$ and was extracted with $3 \times 10 \mathrm{~mL}$ of EtOAc. The combined organic layers were washed with brine, dried $\left(\mathrm{Na}_{2} \mathrm{SO} 4\right)$ and concentrated in vacuo. Purification by flash chromatography (EtOAc/hexanes) provided both C4substituted and C2-substituted products.

IV. General procedure for the Pd-catalyzed amination of 6-aryl-2,4-dichloropyrimidines with aliphatic secondary amines: A round-bottom flask was charged with 6-aryl-2,4-dichloropyrimidine (1.00 mmol), $\mathrm{Pd}(\mathrm{OAc})_{2}(0.02 \mathrm{mmol})$, dppb $(0.02 \mathrm{mmol})$ (or compound 6, $\left.0.02 \mathrm{mmol}\right)$, and $5 \mathrm{~mL}$ of THF. The flask was capped and subjected to three cycles of evacuation-backfilling with nitrogen. The mixture was cooled to $0{ }^{\circ} \mathrm{C}$ or $-20{ }^{\circ} \mathrm{C}$. To this cooled solution was added dropwise the mixture of LiHMDS (1 M in THF, $1.10 \mathrm{mmol}$ ) and amine $(1.00 \mathrm{mmol})$. After the reaction was completed as indicated by HPLC, $10 \mathrm{~mL}$ of water was added to the reaction. The mixture was extracted with $3 \times 10 \mathrm{~mL}$ of EtOAc. The combined organic layers were washed with brine, dried $\left(\mathrm{Na}_{2} \mathrm{SO} 4\right)$ and concentrated in vacuo. Purification by flash chromatography (EtOAc/hexanes) provided the desired product.

V. General procedure for the nucleophilic aromatic substitution of 6-aryl-2,4-dichloropyrimidines with anilide: A solution of 6-aryl-2,4-dichloropyrimidine $(1.00 \mathrm{mmol})$ with aniline or $\mathrm{N}$-methylaniline $(1.00 \mathrm{mmol})$ in $5 \mathrm{~mL}$ of THF was cooled to $-60{ }^{\circ} \mathrm{C}$. To this solution was added dropwise LiHMDS (1.0 M in THF, 2.10 mmol for aniline or $1.10 \mathrm{mmol}$ for $\mathrm{N}$-methylaniline). After $5 \mathrm{~min}, 10 \mathrm{~mL}$ of water was added and the mixture was extracted with $3 \times 10 \mathrm{~mL}$ of EtOAc. The combined organic layers were washed with brine, dried $\left(\mathrm{Na}_{2} \mathrm{SO} 4\right)$ and concentrated in vacuo. Purification by flash chromatography (EtOAc/hexanes) provided desired product.

\section{Analytic data of C4- and C2-amination products:}

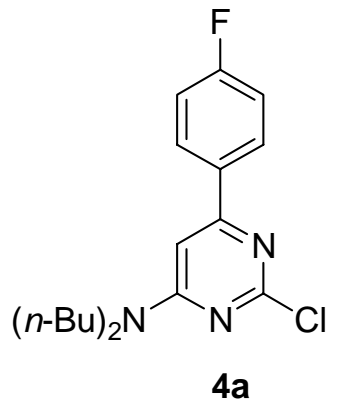

Dibutyl-[2-chloro-6-(4-fluorophenyl)-pyrimidin-4-yl]amine (4a). colorless oil: ${ }^{1} \mathrm{H}$ NMR $\left(\mathrm{CDCl}_{3}, 400 \mathrm{MHz}\right)$ $\delta 7.93(\mathrm{~m}, 2 \mathrm{H}), 7.13(\mathrm{~m}, 2 \mathrm{H}), 6.55(\mathrm{~s}, 1 \mathrm{H}), 3.49(\mathrm{br}, 4 \mathrm{H}), 1.62(\mathrm{~m}, 4 \mathrm{H}), 1.38(\mathrm{~m}, 4 \mathrm{H}), 0.98(\mathrm{t}, J=7.3 \mathrm{~Hz}, 6 \mathrm{H})$; ${ }^{13} \mathrm{C} \mathrm{NMR}\left(\mathrm{CDCl}_{3}, 100 \mathrm{MHz}\right) \delta 164.2(\mathrm{~d}, J=249.9 \mathrm{~Hz}), 163.6,163.0,160.9,133.3(\mathrm{~d}, J=3.2 \mathrm{~Hz}), 129.0(\mathrm{~d}, J=$ 8.2 Hz), 115.6 (d, $J=21.6 \mathrm{~Hz}$ ), 96.1, 48.1, 29.5, 20.1, 13.8; Anal. Calcd for $\mathrm{C}_{18} \mathrm{H}_{23} \mathrm{ClFN}_{3}: \mathrm{C}, 64.37 ; \mathrm{H}, 6.90 ; \mathrm{N}$, 12.51. Found: C, 64.55; H, 6.85; N, 12.61 . 
<smiles>CCCCNc1nc(Cl)cc(-c2ccc(F)cc2)n1</smiles>

$5 a$

Dibutyl-[4-chloro-6-(4-fluorophenyl)-pyrimidin-2-yl]amine (5a). colorless oil: ${ }^{1} \mathrm{H}$ NMR $\left(\mathrm{CDCl}_{3}, 400 \mathrm{MHz}\right)$ $\delta 8.02(\mathrm{~m}, 2 \mathrm{H}), 7.15(\mathrm{~m}, 2 \mathrm{H}), 6.84(\mathrm{~s}, 1 \mathrm{H}), 3.64(\mathrm{br}, 4 \mathrm{H}), 1.66(\mathrm{~m}, 4 \mathrm{H}), 1.40(\mathrm{~m}, 4 \mathrm{H}), 0.99(\mathrm{t}, J=7.3 \mathrm{~Hz}, 6 \mathrm{H})$; ${ }^{13} \mathrm{C} \mathrm{NMR}\left(\mathrm{CDCl}_{3}, 100 \mathrm{MHz}\right) \delta 164.4(\mathrm{~d}, J=250.7 \mathrm{~Hz}), 164.3,161.6,161.4,133.2(\mathrm{~d}, J=3.2 \mathrm{~Hz}), 129.0(\mathrm{~d}, J=$ 8.8 Hz), $115.6(\mathrm{~d}, J=21.6 \mathrm{~Hz}), 103.3,47.6,29.8,20.2$, 14.0; Anal. Calcd for $\mathrm{C}_{18} \mathrm{H}_{23} \mathrm{ClFN}_{3}$ : C, 64.37; H, 6.90; N, 12.51. Found: C, 64.59; H, 6.75; N, 12.60 .<smiles>CN(Cc1ccccc1)c1cc(-c2ccc(F)cc2)nc(Cl)n1</smiles>

$4 b$

Benzyl-[2-chloro-6-(4-fluorophenyl)-pyrimidin-4-yl]methylamine (4b). white solid: mp 95-97 ${ }^{\circ} \mathrm{C} ;{ }^{1} \mathrm{H}$ NMR $\left(\mathrm{CDCl}_{3}, 400 \mathrm{MHz}\right) \delta 7.94(\mathrm{~m}, 2 \mathrm{H}), 7.32(\mathrm{~m}, 3 \mathrm{H}), 7.26(\mathrm{~m}, 2 \mathrm{H}), 7.11(\mathrm{~m}, 2 \mathrm{H}), 6.65(\mathrm{~s}, 1 \mathrm{H}), 4.84(\mathrm{~s}, 2 \mathrm{H}), 3.13(\mathrm{~s}$, $3 \mathrm{H}) ;{ }^{13} \mathrm{C} \mathrm{NMR}\left(\mathrm{CDCl}_{3}, 100 \mathrm{MHz}\right) \delta 164.2(\mathrm{~d}, J=250.5 \mathrm{~Hz}), 164.1,164.0,160.8,132.9(\mathrm{~d}, J=3.2 \mathrm{~Hz}), 129.0$ $(\mathrm{d}, J=8.4 \mathrm{~Hz}), 128.8,127.6,127.3,127.2,115.6(\mathrm{~d}, J=21.8 \mathrm{~Hz}), 96.0,52.8,35.7$; Anal. Calcd for $\mathrm{C}_{18} \mathrm{H}_{15} \mathrm{ClFN}_{3}$ : C, 65.96; H, 4.61; N, 12.82. Found: C, 65.87; H, 4.35; N, 12.62.<smiles>CN(Cc1ccccc1)c1nc(Cl)cc(-c2ccc(F)cc2)n1</smiles>

$5 b$

Benzyl-[4-chloro-6-(4-fluorophenyl)-pyrimidin-2-yl]methylamine (5b). white solid: mp 90-92 ${ }^{\circ} \mathrm{C} ;{ }^{1} \mathrm{H}$ NMR $\left(\mathrm{CDCl}_{3}, 400 \mathrm{MHz}\right) \delta 8.04(\mathrm{~m}, 2 \mathrm{H}), 7.35(\mathrm{~m}, 5 \mathrm{H}), 7.16(\mathrm{~m}, 2 \mathrm{H}), 6.95(\mathrm{~s}, 1 \mathrm{H}), 5.02(\mathrm{~s}, 2 \mathrm{H}), 3.25(\mathrm{~s}, 3 \mathrm{H}) ;{ }^{13} \mathrm{C}$ $\operatorname{NMR}\left(\mathrm{CDCl}_{3}, 100 \mathrm{MHz}\right) \delta 164.5(\mathrm{~d}, J=251.3 \mathrm{~Hz}), 164.6,162.0,161.8,137.9,132.7(\mathrm{~d}, J=3.2 \mathrm{~Hz}), 129.1(\mathrm{~d}$, $J=8.8 \mathrm{~Hz}), 128.5,127.5,127.2,115.6(\mathrm{~d}, J=21.8 \mathrm{~Hz}), 104.1,52.5$, 34.9; Anal. Calcd for $\mathrm{C}_{18} \mathrm{H}_{15} \mathrm{ClFN}_{3}$ : $\mathrm{C}_{\text {, }}$ 65.96; H, 4.61; N, 12.82. Found: C, 65.81; H, 4.35; N, 12.62. 
<smiles>Fc1ccc(-c2cc(N3CCOCC3)nc(Cl)n2)cc1</smiles>

4-[2-Chloro-6-(4-fluorophenyl)-pyrimidin-4-yl]morpholine (4c). white solid: $\mathrm{mp}$ 152-153 ${ }^{\circ} \mathrm{C} ;{ }^{1} \mathrm{H} \mathrm{NMR}$ $\left(\mathrm{CDCl}_{3}, 400 \mathrm{MHz}\right) \delta 7.93(\mathrm{~m}, 2 \mathrm{H}), 7.11(\mathrm{~m}, 2 \mathrm{H}), 6.66(\mathrm{~s}, 1 \mathrm{H}), 3.77(\mathrm{~m}, 4 \mathrm{H}), 3.68(\mathrm{~m}, 4 \mathrm{H}) ;{ }^{13} \mathrm{C} \mathrm{NMR}\left(\mathrm{CDCl}_{3}\right.$, $100 \mathrm{MHz}) \delta 164.4,164.2(\mathrm{~d}, J=250.7 \mathrm{~Hz}), 163.6,160.8,132.6(\mathrm{~d}, J=3.0 \mathrm{~Hz}), 129.0(\mathrm{~d}, J=8.7 \mathrm{~Hz}), 115.6(\mathrm{~d}$, $J=21.8 \mathrm{~Hz}$ ), 96.0, 66.2, 44.3; Anal. Calcd for $\mathrm{C}_{14} \mathrm{H}_{13} \mathrm{ClFN}_{3} \mathrm{O}$ : C, 57.25; H, 4.46; N, 14.31. Found: C, 56.89; H, $4.39 ; \mathrm{N}, 14.08$.<smiles>Fc1ccc(-c2cc(Cl)nc(N3CCOCC3)n2)cc1</smiles>

$5 c$

4-[4-Chloro-6-(4-fluorophenyl)-pyrimidin-2-yl]morpholine (5c). white solid: mp 125-126 ${ }^{\circ} \mathrm{C}$; ${ }^{1} \mathrm{H}$ NMR $\left(\mathrm{CDCl}_{3}, 400 \mathrm{MHz}\right) \delta 8.01(\mathrm{~m}, 2 \mathrm{H}), 7.15(\mathrm{~m}, 2 \mathrm{H}), 6.93(\mathrm{~s}, 1 \mathrm{H}), 3.91(\mathrm{~m}, 4 \mathrm{H}), 3.79(\mathrm{~m}, 4 \mathrm{H}) ;{ }^{13} \mathrm{C} \mathrm{NMR}\left(\mathrm{CDCl}_{3}\right.$, $100 \mathrm{MHz}) \delta 164.9,164.6(\mathrm{~d}, J=250.5 \mathrm{~Hz}), 162.0,161.5,132.7(\mathrm{~d}, J=3.0 \mathrm{~Hz}), 129.2(\mathrm{~d}, J=8.8 \mathrm{~Hz}), 115.7(\mathrm{~d}$, $J=21.9 \mathrm{~Hz}$ ), 104.9, 66.7, 44.4; Anal. Calcd for $\mathrm{C}_{14} \mathrm{H}_{13} \mathrm{ClFN}_{3} \mathrm{O}: \mathrm{C}, 57.25 ; \mathrm{H}, 4.46 ; \mathrm{N}, 14.31$. Found: C, 56.89; $\mathrm{H}$, $4.26 ; \mathrm{N}, 14.05$.<smiles>Fc1ccc(-c2cc(N3CCCCC3)nc(Cl)n2)cc1</smiles>

2-Chloro-4-(4-fluorophenyl)-6-piperidin-1-yl-pyrimidine (4d). colorless oil; ${ }^{1} \mathrm{H} \mathrm{NMR}\left(\mathrm{CDCl}_{3}, 400 \mathrm{MHz}\right) \delta$ $7.91(\mathrm{~m}, 2 \mathrm{H}), 7.09(\mathrm{~m}, 2 \mathrm{H}), 6.66(\mathrm{~s}, 1 \mathrm{H}), 3.65(\mathrm{~m}, 4 \mathrm{H}), 1.69(\mathrm{~m}, 2 \mathrm{H}), 1.63(\mathrm{~m}, 4 \mathrm{H}) ;{ }^{13} \mathrm{C} \mathrm{NMR}\left(\mathrm{CDCl}_{3}, 100\right.$ $\mathrm{MHz}) \delta 164.1(\mathrm{~d}, J=250.5 \mathrm{~Hz}), 164.0,163.1,160.9,133.0(\mathrm{~d}, J=3.2 \mathrm{~Hz}), 128.9(\mathrm{~d}, J=8.7 \mathrm{~Hz}), 115.4(\mathrm{~d}, J=$ 21.7 Hz), 96.1, 45.3, 25.4, 24.3; Anal. Calcd for $\mathrm{C}_{15} \mathrm{H}_{15} \mathrm{ClFN}_{3}$ : C, 61.75; H, 5.18; N, 14.40. Found: C, 61.74; $\mathrm{H}$, $5.07 ; \mathrm{N}, 14.25$. 
<smiles>Fc1ccc(-c2cc(Cl)nc(N3CCCCC3)n2)cc1</smiles>

$5 d$

4-Chloro-6-(4-fluorophenyl)-2-piperidin-1-yl-pyrimidine (5d). white solid: mp 102-103 ${ }^{\circ} \mathrm{C} ;{ }^{1} \mathrm{H} \mathrm{NMR}\left(\mathrm{CDCl}_{3}\right.$, $400 \mathrm{MHz}) \delta 8.01(\mathrm{~m}, 2 \mathrm{H}), 7.14(\mathrm{~m}, 2 \mathrm{H}), 6.82(\mathrm{~s}, 1 \mathrm{H}), 3.87(\mathrm{~m}, 4 \mathrm{H}), 1.70(\mathrm{~m}, 2 \mathrm{H}), 1.63(\mathrm{~m}, 4 \mathrm{H}) ;{ }^{13} \mathrm{C} \mathrm{NMR}$ $\left(\mathrm{CDCl}_{3}, 100 \mathrm{MHz}\right) \delta 164.6,164.4(\mathrm{~d}, J=251.4 \mathrm{~Hz}), 161.8,161.3,132.9(\mathrm{~d}, J=3.2 \mathrm{~Hz}), 129.1(\mathrm{~d}, J=8.8 \mathrm{~Hz})$, $115.6(\mathrm{~d}, J=21.7 \mathrm{~Hz}), 103.6,44.9,25.7,24.7$; Anal. Calcd for $\mathrm{C}_{15} \mathrm{H}_{15} \mathrm{ClFN}_{3}$ : C, 61.75; H, 5.18; N, 14.40 . Found: C, 61.82; H, 5.12; N, 14.34.<smiles>CN1CCN(c2cc(-c3ccc(F)cc3)nc(Cl)n2)CC1</smiles>

2-Chloro-4-(4-fluorophenyl)-6-(4-methylpiperazin-1-yl)pyrimidine (4e). white solid: $\mathrm{mp} \quad 110-112{ }^{\circ} \mathrm{C}$; ${ }^{1} \mathrm{H}$ $\operatorname{NMR}\left(\mathrm{CDCl}_{3}, 400 \mathrm{MHz}\right) \delta 7.87(\mathrm{~m}, 2 \mathrm{H}), 7.05(\mathrm{~m}, 2 \mathrm{H}), 6.43(\mathrm{~s}, 1 \mathrm{H}), 3.67(\mathrm{~m}, 4 \mathrm{H}), 2.43(\mathrm{~m}, 4 \mathrm{H}), 2.29(\mathrm{~s}, 3 \mathrm{H})$; ${ }^{13} \mathrm{C} \mathrm{NMR}\left(\mathrm{CDCl}_{3}, 100 \mathrm{MHz}\right) \delta 164.2,164.1(\mathrm{~d}, J=251.0 \mathrm{~Hz}), 163.3,160.7,132.7(\mathrm{~d}, J=3.2 \mathrm{~Hz}), 128.9(\mathrm{~d}, J=$ $8.8 \mathrm{~Hz}), 115.5(\mathrm{~d}, J=21.7 \mathrm{~Hz}), 96.2,54.3,45.9$, 44.0; Anal. Calcd for $\mathrm{C}_{15} \mathrm{H}_{16} \mathrm{ClFN}_{4}$ : C, 58.73; H, 5.26; N, 18.26. Found: C, 58.58; H, 5.01; N, 17.92 .<smiles>CN1CCN(c2nc(Cl)cc(-c3ccc(F)cc3)n2)CC1</smiles>

4-Chloro-6-(4-fluorophenyl)-2-(4-methylpiperazin-1-yl)pyrimidine (5e). white solid: mp 97-99 ${ }^{\circ} \mathrm{C} ;{ }^{1} \mathrm{H}$ NMR $\left(\mathrm{CDCl}_{3}, 400 \mathrm{MHz}\right) \delta 8.00(\mathrm{~m}, 2 \mathrm{H}), 7.14(\mathrm{~m}, 2 \mathrm{H}), 6.88(\mathrm{~s}, 1 \mathrm{H}), 3.94(\mathrm{~m}, 4 \mathrm{H}), 2.48(\mathrm{~m}, 4 \mathrm{H}), 2.35(\mathrm{~s}, 3 \mathrm{H}) ;{ }^{13} \mathrm{C}$ $\operatorname{NMR}\left(\mathrm{CDCl}_{3}, 100 \mathrm{MHz}\right) \delta 164.8,164.5(\mathrm{~d}, J=251.4 \mathrm{~Hz}), 161.9,161.3,132.7(\mathrm{~d}, J=3.1 \mathrm{~Hz}), 129.1(\mathrm{~d}, J=8.8$ $\mathrm{Hz}), 115.7(\mathrm{~d}, J=21.7 \mathrm{~Hz}), 104.5,54.8,46.2$, 43.8; Anal. Calcd for $\mathrm{C}_{15} \mathrm{H}_{16} \mathrm{ClFN}_{4}$ : C, 58.73; H, 5.26; N, 18.26. Found: C, 58.69; H, 5.02; N, 17.89 . 
<smiles>Fc1ccc(-c2cc(N3CCCC3)nc(Cl)n2)cc1</smiles>

$4 f$

2-Chloro-4-(4-fluorophenyl)-6-pyrrolidin-1-yl-pyrimidine (4f). white solid: mp 120-121 ${ }^{\circ} \mathrm{C}$; ${ }^{1} \mathrm{H}$ NMR $\left(\mathrm{CDCl}_{3}, 400 \mathrm{MHz}\right) \delta 7.91(\mathrm{~m}, 2 \mathrm{H}), 7.08(\mathrm{~m}, 2 \mathrm{H}), 6.40(\mathrm{~s}, 1 \mathrm{H}), 3.58(\mathrm{br}, 2 \mathrm{H}), 3.37(\mathrm{br}, 2 \mathrm{H}), 2.03(\mathrm{br}, 2 \mathrm{H}), 1.96$ $(\mathrm{br}, 2 \mathrm{H}) ;{ }^{13} \mathrm{C} \mathrm{NMR}\left(\mathrm{CDCl}_{3}, 100 \mathrm{MHz}\right) \delta 164.1(\mathrm{~d}, J=250.8 \mathrm{~Hz}), 162.8,161.6,160.6,132.8(\mathrm{~d}, J=3.2 \mathrm{~Hz})$, $128.9(\mathrm{~d}, J=8.2 \mathrm{~Hz}), 115.4(\mathrm{~d}, J=21.6 \mathrm{~Hz}), 96.9,46.8,46.4,25.4,24.7$; Anal. Calcd for $\mathrm{C}_{14} \mathrm{H}_{13} \mathrm{ClFN}_{3}: \mathrm{C}_{\text {, }}$ 60.55; H, 4.72; N, 15.13. Found: C, 60.56; H, 4.60; N, 15.00.<smiles>Fc1ccc(-c2cc(Cl)nc(N3CCCC3)n2)cc1</smiles>

$5 f$

4-Chloro-6-(4-fluorophenyl)-2-pyrrolidin-1-yl-pyrimidine (5f). white solid: $\mathrm{mp}$ 121-122 ${ }^{\circ} \mathrm{C}$; ${ }^{1} \mathrm{H}$ NMR $\left(\mathrm{CDCl}_{3}, 400 \mathrm{MHz}\right) \delta 8.03(\mathrm{~m}, 2 \mathrm{H}), 7.13(\mathrm{~m}, 2 \mathrm{H}), 6.85(\mathrm{~s}, 1 \mathrm{H}), 3.65(\mathrm{br}, 4 \mathrm{H}), 2.00(\mathrm{~m}, 4 \mathrm{H}) ;{ }^{13} \mathrm{C} \mathrm{NMR}\left(\mathrm{CDCl}_{3}\right.$, $100 \mathrm{MHz}) \delta 164.5,164.4(\mathrm{~d}, J=251.3 \mathrm{~Hz}), 161.5,160.1,132.9(\mathrm{~d}, J=3.2 \mathrm{~Hz}), 129.1(\mathrm{~d}, J=8.8 \mathrm{~Hz}), 115.6(\mathrm{~d}$, $J=21.7 \mathrm{~Hz}$ ), 103.6, 46.8, 25.4; Anal. Calcd for $\mathrm{C}_{14} \mathrm{H}_{13} \mathrm{ClFN}_{3}$ : C, 60.55; H, 4.72; N, 15.13. Found: C, 60.47; H, $4.68 ; \mathrm{N}, 14.97$.<smiles>CC1CCCN1c1cc(-c2ccc(F)cc2)nc(Cl)n1</smiles>

$4 \mathrm{~g}$

2-Chloro-4-(4-fluorophenyl)-6-(2-methylpyrrolidin-1-yl)pyrimidine (4g). colorless oil: ${ }^{1} \mathrm{H}$ NMR $\left(\mathrm{CDCl}_{3}\right.$, $400 \mathrm{MHz}) \delta 7.95(\mathrm{~m}, 2 \mathrm{H}), 7.12(\mathrm{~m}, 2 \mathrm{H}), 6.47(\mathrm{~s}, 1 \mathrm{H}), 4.45(\mathrm{br}, 1 \mathrm{H}), 3.57(\mathrm{br}, 2 \mathrm{H}), 2.11$ (br, 3H), $1.77(\mathrm{br}, 1 \mathrm{H})$, $1.27(\mathrm{~d}, J=6.3 \mathrm{~Hz}, 3 \mathrm{H}) ;{ }^{13} \mathrm{C} \mathrm{NMR}\left(\mathrm{CDCl}_{3}, 100 \mathrm{MHz}\right) \delta 164.1(\mathrm{~d}, J=250.5 \mathrm{~Hz}), 163.0,161.3,160.7,132.9(\mathrm{~d}$, $J=3.2 \mathrm{~Hz}), 128.9(\mathrm{~d}, J=8.3 \mathrm{~Hz}), 115.4(\mathrm{~d}, J=21.7 \mathrm{~Hz}), 97.1,53.5,46.9,32.1,22.8,18.7$; Anal. Calcd for $\mathrm{C}_{15} \mathrm{H}_{15} \mathrm{ClFN}_{3}$ : C, 61.75; H, 5.18; N, 14.40. Found: C, 61.44; H, 5.05; N, 14.27. 
<smiles>CC1CCCN1c1nc(Cl)cc(-c2ccc(F)cc2)n1</smiles>

$5 g$

4-Chloro-6-(4-fluorophenyl)-2-(2-methylpyrrolidin-1-yl)pyrimidine (5g). colorless oil: ${ }^{1} \mathrm{H} \mathrm{NMR}_{(\mathrm{CDCl}}$, $400 \mathrm{MHz}) \delta 8.02(\mathrm{~m}, 2 \mathrm{H}), 7.13(\mathrm{~m}, 2 \mathrm{H}), 6.85(\mathrm{~s}, 1 \mathrm{H}), 4.37(\mathrm{br}, 1 \mathrm{H}), 3.71(\mathrm{br}, 1 \mathrm{H}), 3.63(\mathrm{br}, 1 \mathrm{H}), 2.09(\mathrm{~m}, 2 \mathrm{H})$, $1.96(\mathrm{~m}, 1 \mathrm{H}), 1.74(\mathrm{~m}, 1 \mathrm{H}), 1.31(\mathrm{~d}, J=5.8 \mathrm{~Hz}, 3 \mathrm{H}) ;{ }^{13} \mathrm{C} \mathrm{NMR}\left(\mathrm{CDCl}_{3}, 100 \mathrm{MHz}\right) \delta 164.4(\mathrm{~d}, J=250.5 \mathrm{~Hz})$, 164.3, 161.5, 159.9, $133.0(\mathrm{~d}, J=3.2 \mathrm{~Hz}), 129.0(\mathrm{~d}, J=8.8 \mathrm{~Hz}), 115.5(\mathrm{~d}, J=21.6 \mathrm{~Hz}), 103.5,53.5,47.2,32.8$, 23.2, 19.3; Anal. Calcd for $\mathrm{C}_{15} \mathrm{H}_{15} \mathrm{ClFN}_{3}$ : C, 61.75; H, 5.18; N, 14.40. Found: C, 61.55; H, 5.03; N, 14.32.<smiles>Fc1ccc(-c2cc(Nc3ccccc3)nc(Cl)n2)cc1</smiles>

4h

[2-Chloro-6-(4-fluorophenyl)-pyrimidin-4-yl]-phenylamine (4h). white solid: mp 132-133 ${ }^{\circ} \mathrm{C}$; ${ }^{1} \mathrm{H}$ NMR $\left(\mathrm{CDCl}_{3}, 400 \mathrm{MHz}\right) \delta 7.89(\mathrm{~m}, 2 \mathrm{H}), 7.82(\mathrm{br}, 1 \mathrm{H}), 7.44(\mathrm{~m}, 2 \mathrm{H}), 7.37(\mathrm{~m}, 2 \mathrm{H}), 7.27(\mathrm{~m}, 1 \mathrm{H}), 7.09(\mathrm{~m}, 2 \mathrm{H}), 6.88$ $(\mathrm{s}, 1 \mathrm{H}) ;{ }^{13} \mathrm{C} \mathrm{NMR}\left(\mathrm{CDCl}_{3}, 100 \mathrm{MHz}\right) \delta 164.9,164.5(\mathrm{~d}, J=251.5 \mathrm{~Hz}), 163.5,160.8,137.2,132.1(\mathrm{~d}, J=3.2$ $\mathrm{Hz}), 129.6,129.1(\mathrm{~d}, J=8.8 \mathrm{~Hz}), 126.0,123.6,115.7(\mathrm{~d}, J=21.9 \mathrm{~Hz}), 97.1$; Anal. Calcd for $\mathrm{C}_{16} \mathrm{H}_{11} \mathrm{ClFN}_{3}$ : C, 64.11; H, 3.70; N, 14.02. Found: C, 63.94; H, 3.50; N, 13.96.<smiles>Fc1ccc(-c2cc(Cl)nc(Nc3ccccc3)n2)cc1</smiles>

$5 \mathrm{~h}$

[4-Chloro-6-(4-fluorophenyl)-pyrimidin-2-yl]-phenylamine (5h). white solid: mp 145-147 ${ }^{\circ} \mathrm{C} ;{ }^{1} \mathrm{H}$ NMR $\left(\mathrm{CDCl}_{3}, 400 \mathrm{MHz}\right) \delta 8.05(\mathrm{~m}, 2 \mathrm{H}), 7.66(\mathrm{~d}, J=7.9 \mathrm{~Hz}, 2 \mathrm{H}), 7.39(\mathrm{t}, J=7.7 \mathrm{~Hz}, 2 \mathrm{H}), 7.29(\mathrm{br}, 1 \mathrm{H}), 7.19(\mathrm{~m}$, $2 \mathrm{H}), 7.13(\mathrm{~s}, 1 \mathrm{H}), 7.11(\mathrm{~m}, 1 \mathrm{H}) ;{ }^{13} \mathrm{C} \mathrm{NMR}\left(\mathrm{CDCl}_{3}, 100 \mathrm{MHz}\right) \delta 165.4,164.8(\mathrm{~d}, J=252.2 \mathrm{~Hz}), 161.9,159.6$, 138.7, $132.2(\mathrm{~d}, J=3.0 \mathrm{~Hz}), 129.3(\mathrm{~d}, J=8.2 \mathrm{~Hz}), 129.0,123.1,119.5,115.9$ (d, $J=21.7 \mathrm{~Hz})$, 107.5 ; Anal. Calcd for $\mathrm{C}_{16} \mathrm{H}_{11} \mathrm{ClFN}_{3}$ : C, 64.11; H, 3.70; N, 14.02. Found: C, 63.71; H, 3.57; N, 13.84 . 
<smiles>CN(c1ccccc1)c1cc(-c2ccc(F)cc2)nc(Cl)n1</smiles>

[2-Chloro-6-(4-fluorophenyl)-pyrimidin-4-yl]methylphenylamine (4i). white solid: $\mathrm{mp} \quad{ }^{133-134}{ }^{\circ} \mathrm{C}$; ${ }^{1} \mathrm{H}$ NMR $\left(\mathrm{CDCl}_{3}, 400 \mathrm{MHz}\right) \delta 7.78(\mathrm{~m}, 2 \mathrm{H}), 7.53(\mathrm{~m}, 2 \mathrm{H}), 7.41(\mathrm{~m}, 1 \mathrm{H}), 7.29(\mathrm{~m}, 2 \mathrm{H}), 7.03(\mathrm{~m}, 2 \mathrm{H}), 6.47(\mathrm{~s}, 1 \mathrm{H})$, $3.52(\mathrm{~s}, 3 \mathrm{H}) ;{ }^{13} \mathrm{C} \mathrm{NMR}\left(\mathrm{CDCl}_{3}, 100 \mathrm{MHz}\right) \delta 164.3,164.1(\mathrm{~d}, J=250.2 \mathrm{~Hz}), 163.1,160.9,143.6,132.5(\mathrm{~d}, J=$ $3.2 \mathrm{~Hz}), 130.3,129.0(\mathrm{~d}, J=8.8 \mathrm{~Hz}), 127.8,126.8,115.5(\mathrm{~d}, J=21.9 \mathrm{~Hz}), 98.1,38.5$; Anal. Calcd for $\mathrm{C}_{17} \mathrm{H}_{13} \mathrm{ClFN}_{3}$ : C, 65.08; H, 4.18; N, 13.39. Found: C, 64.82; H, 3.90; N, 13.22.<smiles>CN(c1ccccc1)c1nc(Cl)cc(-c2ccc(F)cc2)n1</smiles>

[4-Chloro-6-(4-fluorophenyl)-pyrimidin-2-yl]methylphenylamine (5i). white solid: $\mathrm{mp} \quad 115-116{ }^{\circ} \mathrm{C}$; ${ }^{1} \mathrm{H}$ NMR $\left(\mathrm{CDCl}_{3}, 400 \mathrm{MHz}\right) \delta 7.93(\mathrm{~m}, 2 \mathrm{H}), 7.47(\mathrm{~m}, 2 \mathrm{H}), 7.42(\mathrm{~m}, 2 \mathrm{H}), 7.28(\mathrm{~m}, 1 \mathrm{H}), 7.10(\mathrm{~m}, 2 \mathrm{H}), 7.02(\mathrm{~s}, 1 \mathrm{H})$, $3.64(\mathrm{~s}, 3 \mathrm{H}) ;{ }^{13} \mathrm{C} \mathrm{NMR}\left(\mathrm{CDCl}_{3}, 100 \mathrm{MHz}\right) \delta 164.5(\mathrm{~d}, J=251.4 \mathrm{~Hz}), 164.4,161.7,161.5,144.8,132.5(\mathrm{~d}, J=$ $3.0 \mathrm{~Hz}), 129.2(\mathrm{~d}, J=8.8 \mathrm{~Hz}), 128.8,126.3,125.8,115.7(\mathrm{~d}, J=21.7 \mathrm{~Hz}), 105.4,38.7$; Anal. Calcd for $\mathrm{C}_{17} \mathrm{H}_{13} \mathrm{ClFN}_{3}$ : C, 65.08; H, 4.18; N, 13.39. Found: C, 64.93; H, 3.91; N, 13.23.<smiles>CCCCCNc1cc(-c2ccccc2)nc(Cl)n1</smiles>

8 a

Dibutyl-(2-chloro-6-phenylpyrimidin-4-yl)amine (8a). colorless oil: ${ }^{1} \mathrm{H}$ NMR $\left(\mathrm{CDCl}_{3}, 400 \mathrm{MHz}\right) \delta 7.93(\mathrm{~m}$, 2H), $7.46(\mathrm{~m}, 3 \mathrm{H}), 6.61(\mathrm{~s}, 1 \mathrm{H}), 3.50(\mathrm{br}, 4 \mathrm{H}), 1.62(\mathrm{~m}, 4 \mathrm{H}), 1.39(\mathrm{~m}, 4 \mathrm{H}), 0.99(\mathrm{t}, J=7.3 \mathrm{~Hz}, 6 \mathrm{H}) ;{ }^{13} \mathrm{C} \mathrm{NMR}$ $\left(\mathrm{CDCl}_{3}, 100 \mathrm{MHz}\right) \delta 164.7,163.0,160.9,137.1,130.2,128.6,127.0,96.5,48.1,29.4,20.1$, 13.9; Anal. Calcd for $\mathrm{C}_{18} \mathrm{H}_{24} \mathrm{ClN}_{3}$ : C, 68.02; H, 7.61; N, 13.22. Found: C, 68.05; H, 7.81; N, 13.11 .<smiles>CCCCNc1nc(Cl)cc(-c2ccccc2)n1</smiles>

9a

Dibutyl-(4-chloro-6-phenylpyrimidin-2-yl)amine (9a). colorless oil: ${ }^{1} \mathrm{H}$ NMR $\left(\mathrm{CDCl}_{3}, 400 \mathrm{MHz}\right) \delta 8.05(\mathrm{~m}$, 2H), $7.48(\mathrm{~m}, 3 \mathrm{H}), 6.92(\mathrm{~s}, 1 \mathrm{H}), 3.67(\mathrm{br}, 4 \mathrm{H}), 1.68(\mathrm{~m}, 4 \mathrm{H}), 1.42(\mathrm{~m}, 4 \mathrm{H}), 1.01(\mathrm{t}, J=7.3 \mathrm{~Hz}, 6 \mathrm{H}) ;{ }^{13} \mathrm{C} \mathrm{NMR}$ $\left(\mathrm{CDCl}_{3}, 100 \mathrm{MHz}\right) \delta 165.4,161.5,162.4,137.0,130.6,128.6,127.0,103.6,47.5,29.8,20.2$, 14.0; Anal. Calcd for $\mathrm{C}_{18} \mathrm{H}_{24} \mathrm{ClN}_{3}$ : C, 68.02; H, 7.61; N, 13.22. Found: C, 67.95; H, 7.70; N, 13.09. 
<smiles>Clc1nc(-c2ccccc2)cc(N2CCOCC2)n1</smiles>

$8 b$

4-(2-Chloro-6-phenyl-pyrimidin-4-yl)morpholine (8b). white solid: mp 161-162 ${ }^{\circ} \mathrm{C} ;{ }^{1} \mathrm{H}$ NMR $\left(\mathrm{CDCl}_{3}, 400\right.$ $\mathrm{MHz}) \delta 7.91(\mathrm{~m}, 2 \mathrm{H}), 7.44(\mathrm{~m}, 3 \mathrm{H}), 6.70(\mathrm{~s}, 1 \mathrm{H}), 3.81(\mathrm{~m}, 4 \mathrm{H}), 3.66(\mathrm{~m}, 4 \mathrm{H}) ;{ }^{13} \mathrm{C} \mathrm{NMR}\left(\mathrm{CDCl}_{3}, 100 \mathrm{MHz}\right) \delta$ 165.5, 163.6, 160.8, 136.4, 130.5, 128.6, 126.9, 96.5, 66.2, 44.3; Anal. Calcd for $\mathrm{C}_{14} \mathrm{H}_{14} \mathrm{ClN}_{3} \mathrm{O}: \mathrm{C}, 60.98$; $\mathrm{H}$, 5.12; N, 15.24. Found: C, 61.02; H, 4.92; N, 14.98.<smiles>Clc1cc(-c2ccccc2)nc(N2CCOCC2)n1</smiles>

$9 b$

4-(4-Chloro-6-phenylpyrimidin-2-yl)morpholine (9b). white solid: mp 90-91 ${ }^{\circ} \mathrm{C} ;{ }^{1} \mathrm{H} \mathrm{NMR}\left(\mathrm{CDCl}_{3}, 400 \mathrm{MHz}\right)$ $\delta 8.00(\mathrm{~m}, 2 \mathrm{H}), 7.46(\mathrm{~m}, 3 \mathrm{H}), 6.97(\mathrm{~s}, 1 \mathrm{H}), 3.91(\mathrm{~m}, 4 \mathrm{H}), 3.78(\mathrm{~m}, 4 \mathrm{H}) ;{ }^{13} \mathrm{C} \mathrm{NMR}\left(\mathrm{CDCl}_{3}, 100 \mathrm{MHz}\right) \delta 165.9$, 161.8, 161.4, 136.4, 130.9, 128.6, 127.0, 105.2, 66.7, 44.2; Anal. Calcd for $\mathrm{C}_{14} \mathrm{H}_{14} \mathrm{ClN}_{3} \mathrm{O}$ : C, 60.98; H, 5.12; N, 15.24. Found: C, 60.81; H, 4.85; N, 15.03 .<smiles>Clc1nc(-c2ccccc2)cc(N2CCCCC2)n1</smiles>

2-Chloro-4-phenyl-6-piperidin-1-yl-pyrimidine (8c). white solid: mp 115-116 ${ }^{\circ} \mathrm{C} ;{ }^{1} \mathrm{H}$ NMR $\left(\mathrm{CDCl}_{3}, 400\right.$ MHz) $\delta 7.95(\mathrm{~m}, 2 \mathrm{H}), 7.44(\mathrm{~m}, 3 \mathrm{H}), 6.74(\mathrm{~s}, 1 \mathrm{H}), 3.68(\mathrm{~m}, 4 \mathrm{H}), 1.71(\mathrm{~m}, 2 \mathrm{H}), 1.64(\mathrm{~m}, 4 \mathrm{H}) ;{ }^{13} \mathrm{C} \mathrm{NMR}\left(\mathrm{CDCl}_{3}\right.$, $100 \mathrm{MHz}) \delta 165.2$, 163.2, 161.0, 136.9, 130.3, 128.6, 126.9, 96.6, 45.4, 25.5, 24.4; Anal. Calcd for $\mathrm{C}_{15} \mathrm{H}_{16} \mathrm{ClN}_{3}$ : C, 65.81; H, 5.89; N, 15.35. Found: C, 65.85; H, 5.81; N, 15.32.<smiles>Clc1cc(-c2ccccc2)nc(N2CCCCC2)n1</smiles>

4-Chloro-6-phenyl-2-piperidin-1-yl-pyrimidine (9c). white solid: mp 95-96 ${ }^{\circ} \mathrm{C} ;{ }^{1} \mathrm{H}$ NMR $\left(\mathrm{CDCl}_{3}, 400 \mathrm{MHz}\right)$ $\delta 8.03(\mathrm{~m}, 2 \mathrm{H}), 7.48(\mathrm{~m}, 3 \mathrm{H}), 6.90(\mathrm{~s}, 1 \mathrm{H}), 3.90(\mathrm{~m}, 4 \mathrm{H}), 1.68(\mathrm{~m}, 6 \mathrm{H}) ;{ }^{13} \mathrm{C} \mathrm{NMR}\left(\mathrm{CDCl}_{3}, 100 \mathrm{MHz}\right) \delta 165.8$, 161.7, 161.4, 136.8, 130.7, 128.6, 127.1, 104.0, 44.9, 25.7, 24.7; Anal. Calcd for $\mathrm{C}_{15} \mathrm{H}_{16} \mathrm{ClN}_{3}$ : C, 65.81; H, 5.89; N, 15.35. Found: C, 65.72; H, 5.76; N, 15.22. 
<smiles>CN(c1ccccc1)c1cc(-c2ccccc2)nc(Cl)n1</smiles>

$8 d$

(2-Chloro-6-phenylpyrimidin-4-yl)methylphenylamine (8d). white solid: mp 135-137 ${ }^{\circ} \mathrm{C} ;{ }^{1} \mathrm{H} \mathrm{NMR}\left(\mathrm{CDCl}_{3}\right.$, $400 \mathrm{MHz}) \delta 7.29(\mathrm{~m}, 2 \mathrm{H}), 7.52(\mathrm{~m}, 2 \mathrm{H}), 7.37(\mathrm{~m}, 4 \mathrm{H}), 7.29(\mathrm{~m}, 2 \mathrm{H}), 6.54(\mathrm{~s}, 1 \mathrm{H}), 3.54(\mathrm{~s}, 3 \mathrm{H}) ;{ }^{13} \mathrm{C} \mathrm{NMR}$ $\left(\mathrm{CDCl}_{3}, 100 \mathrm{MHz}\right) \delta 164.28,164.26,160.8,143.7,136.4,130.4,130.2,128.5,127.7,126.9,126.7,98.6,38.4$; Anal. Calcd for $\mathrm{C}_{17} \mathrm{H}_{14} \mathrm{ClN}_{3}$ : C, 69.03; H, 4.77; N, 14.21. Found: C, 68.85; H, 4.75; N, 14.10.<smiles>CN(c1ccccc1)c1nc(Cl)cc(-c2ccccc2)n1</smiles>

9d

(4-Chloro-6-phenylpyrimidin-2-yl)methylphenylamine (9d). white solid: mp $135-137{ }^{\circ} \mathrm{C}$; ${ }^{1} \mathrm{H} \mathrm{NMR}\left(\mathrm{CDCl}_{3}\right.$, $400 \mathrm{MHz}) \delta 7.96(\mathrm{~m}, 2 \mathrm{H}), 7.44(\mathrm{~m}, 7 \mathrm{H}), 7.27(\mathrm{~m}, 1 \mathrm{H}), 7.09(\mathrm{~s}, 1 \mathrm{H}), 3.65(\mathrm{~s}, 3 \mathrm{H}) ;{ }^{13} \mathrm{C} \mathrm{NMR}\left(\mathrm{CDCl}_{3}, 100 \mathrm{MHz}\right)$ $\delta$ 165.6, 161.62, 161.56, 144.8, 136.4, 130.9, 128.8, 128.7, 127.1, 126.3, 125.7, 105.8, 38.7; Anal. Calcd for $\mathrm{C}_{17} \mathrm{H}_{14} \mathrm{ClN}_{3}$ : C, 69.03; H, 4.77; N, 14.21. Found: C, 68.88; H, 4.77; N, 14.06.<smiles>CCCCCCCCCCCCCCCCCCCC</smiles>

Dibutyl-[2-chloro-6-(4-methoxyphenyl)-pyrimidin-4-yl]amine (11a). colorless oil: ${ }^{1} \mathrm{H}$ NMR $\left(\mathrm{CDCl}_{3}, 400\right.$ $\mathrm{MHz}) \delta 7.90(\mathrm{~d}, J=8.8 \mathrm{~Hz}, 2 \mathrm{H}), 6.93(\mathrm{~d}, J=8.8 \mathrm{~Hz}, 2 \mathrm{H}), 6.53(\mathrm{~s}, 1 \mathrm{H}), 3.82(\mathrm{~s}, 3 \mathrm{H}), 3.46(\mathrm{br}, 4 \mathrm{H}), 1.59(\mathrm{~m}, 4 \mathrm{H})$, $1.36(\mathrm{~m}, 4 \mathrm{H}), 0.96(\mathrm{t}, J=7.3 \mathrm{~Hz}, 6 \mathrm{H}) ;{ }^{13} \mathrm{C} \mathrm{NMR}\left(\mathrm{CDCl}_{3}, 100 \mathrm{MHz}\right) \delta 164.1,162.9,161.4,160.7,129.3,128.3$, 113.8, 95.2, 55.2, 48.0, 29.4, 20.0, 13.8; Anal. Calcd for $\mathrm{C}_{19} \mathrm{H}_{26} \mathrm{ClN}_{3} \mathrm{O}: \mathrm{C}, 65.60 ; \mathrm{H}, 7.53$; N, 12.08. Found: C, 65.48; H, 7.53; N, 11.76.

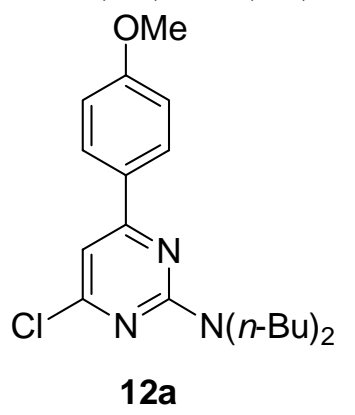

Dibutyl-[4-chloro-6-(4-methoxyphenyl)-pyrimidin-2-yl]amine (12a). colorless oil: ${ }^{1} \mathrm{H}$ NMR $\left(\mathrm{CDCl}_{3}, 400\right.$ $\mathrm{MHz}) \delta 8.00(\mathrm{~m}, 2 \mathrm{H}), 6.98(\mathrm{~m}, 2 \mathrm{H}), 6.84(\mathrm{~s}, 1 \mathrm{H}), 3.87(\mathrm{~s}, 3 \mathrm{H}), 3.65(\mathrm{br}, 4 \mathrm{H}), 1.66(\mathrm{~m}, 4 \mathrm{H}), 1.41(\mathrm{~m}, 4 \mathrm{H}), 1.00(\mathrm{t}$, $J=7.3 \mathrm{~Hz}, 6 \mathrm{H}) ;{ }^{13} \mathrm{C} \mathrm{NMR}\left(\mathrm{CDCl}_{3}, 100 \mathrm{MHz}\right) \delta 164.9,161.7,161.34,161.28,129.4,128.5,113.9,102.8,55.3$, 
47.5, 29.8, 20.2, 14.1; Anal. Calcd for $\mathrm{C}_{19} \mathrm{H}_{26} \mathrm{ClN}_{3} \mathrm{O}: \mathrm{C}, 65.60 ; \mathrm{H}, 7.53 ; \mathrm{N}, 12.08$. Found: $\mathrm{C}, 65.70 ; \mathrm{H}, 7.66 ; \mathrm{N}$, 12.05 .<smiles>COc1ccc(-c2cc(N3CCOCC3)nc(Cl)n2)cc1</smiles>

4-[2-Chloro-6-(4-methoxyphenyl)-pyrimidin-4-yl]morpholine (11b). white solid: mp 156-158 ${ }^{\circ} \mathrm{C} ;{ }^{1} \mathrm{H}$ NMR $\left(\mathrm{CDCl}_{3}, 400 \mathrm{MHz}\right) \delta 7.92(\mathrm{~m}, 2 \mathrm{H}), 6.95(\mathrm{~m}, 2 \mathrm{H}), 6.68(\mathrm{~s}, 1 \mathrm{H}), 3.86(\mathrm{~s}, 3 \mathrm{H}), 3.80(\mathrm{~m}, 4 \mathrm{H}), 3.69(\mathrm{~m}, 4 \mathrm{H}) ;{ }^{13} \mathrm{C}$ $\mathrm{NMR}\left(\mathrm{CDCl}_{3}, 100 \mathrm{MHz}\right) \delta 165.2,163.8,161.8,160.8,128.9,128.6,114.0,95.3,66.4,55.4,44.4$; Anal. Calcd for $\mathrm{C}_{15} \mathrm{H}_{16} \mathrm{ClN}_{3} \mathrm{O}_{2}$ : C, 58.92; H, 5.27; N, 13.74. Found: C, 59.12; H, 5.26; N, 13.71 .<smiles>COc1ccc(-c2cc(Cl)nc(N3CCOCC3)n2)cc1</smiles>

4-[4-Chloro-6-(4-methoxyphenyl)-pyrimidin-2-yl]morpholine (12b). white solid: mp 156-158 ${ }^{\circ} \mathrm{C} ;{ }^{1} \mathrm{H}$ NMR $\left(\mathrm{CDCl}_{3}, 400 \mathrm{MHz}\right) \delta 7.97(\mathrm{~d}, J=8.7 \mathrm{~Hz}, 2 \mathrm{H}), 6.96(\mathrm{~d}, J=8.7 \mathrm{~Hz}, 2 \mathrm{H}), 6.91(\mathrm{~s}, 1 \mathrm{H}), 3.89(\mathrm{~m}, 4 \mathrm{H}), 3.86(\mathrm{~s}, 3 \mathrm{H})$, $3.78(\mathrm{~m}, 4 \mathrm{H}) ;{ }^{13} \mathrm{C}$ NMR $\left(\mathrm{CDCl}_{3}, 100 \mathrm{MHz}\right) \delta 165.4,162.0,161.6,161.4,128.8,128.6,114.0,104.3,66.7,55.3$, 44.3; Anal. Calcd for $\mathrm{C}_{15} \mathrm{H}_{16} \mathrm{ClN}_{3} \mathrm{O}_{2}$ : C, 58.92; H, 5.27; N, 13.74. Found: C, 59.13; H, 5.28; N, 13.66.<smiles>COc1ccc(-c2cc(N3CCCCC3)nc(Cl)n2)cc1</smiles>

2-Chloro-4-(4-methoxyphenyl)-6-piperidin-1-yl-pyrimidine (11c). white solid: mp 156-158 ${ }^{\circ} \mathrm{C} ;{ }^{1} \mathrm{H}$ NMR $\left(\mathrm{CDCl}_{3}, 400 \mathrm{MHz}\right) \delta 7.90(\mathrm{~m}, 2 \mathrm{H}), 6.91(\mathrm{~m}, 2 \mathrm{H}), 6.65(\mathrm{~s}, 1 \mathrm{H}), 3.82(\mathrm{~s}, 3 \mathrm{H}), 3.63(\mathrm{~m}, 4 \mathrm{H}), 1.67(\mathrm{~m}, 2 \mathrm{H}), 1.61(\mathrm{~m}$, $4 \mathrm{H}) ;{ }^{13} \mathrm{C} \mathrm{NMR}\left(\mathrm{CDCl}_{3}, 100 \mathrm{MHz}\right) \delta 164.6,163.1,161.4,160.8,129.2,128.3,113.8,95.3,55.2,45.3,25.4,24.3$; Anal. Calcd for $\mathrm{C}_{16} \mathrm{H}_{18} \mathrm{ClN}_{3} \mathrm{O}$ : C, 63.26; H, 5.97; N, 13.83. Found: C, 63.11; H, 5.83; N, 13.54 . 
<smiles>COc1ccc(-c2cc(Cl)nc(N3CCCCC3)n2)cc1</smiles>

4-Chloro-6-(4-methoxyphenyl)-2-piperidin-1-yl-pyrimidine (12c). white solid: mp 123-124 ${ }^{\circ} \mathrm{C} ;{ }^{1} \mathrm{H}$ NMR $\left(\mathrm{CDCl}_{3}, 400 \mathrm{MHz}\right) \delta 7.99(\mathrm{~m}, 2 \mathrm{H}), 6.97(\mathrm{~m}, 2 \mathrm{H}), 6.83(\mathrm{~s}, 1 \mathrm{H}), 3.89(\mathrm{~m}, 4 \mathrm{H}), 3.87(\mathrm{~s}, 3 \mathrm{H}), 1.67(\mathrm{~m}, 6 \mathrm{H}) ;{ }^{13} \mathrm{C}$ NMR $\left(\mathrm{CDCl}_{3}, 100 \mathrm{MHz}\right) \delta 165.3,161.8,161.5,161.3,129.3,128.6,113.9,103.2,55.3,44.9$, 25.8, 24.8 ; Anal. Calcd for $\mathrm{C}_{16} \mathrm{H}_{18} \mathrm{ClN}_{3} \mathrm{O}: \mathrm{C}, 63.26 ; \mathrm{H}, 5.97 ; \mathrm{N}, 13.83$. Found: C, 63.29; H, 5.87; N, 13.53 .<smiles>COc1ccc(-c2cc(N(C)c3ccccc3)nc(Cl)n2)cc1</smiles>

[2-Chloro-6-(4-methoxyphenyl)-pyrimidin-4-yl]methylphenylamine (11d). white solid: $\mathrm{mp} 125-126{ }^{\circ} \mathrm{C} ;{ }^{1} \mathrm{H}$ $\operatorname{NMR}\left(\mathrm{CDCl}_{3}, 400 \mathrm{MHz}\right) \delta 7.74(\mathrm{~m}, 2 \mathrm{H}), 7.51(\mathrm{~m}, 2 \mathrm{H}), 7.39(\mathrm{~m}, 1 \mathrm{H}), 7.28(\mathrm{~m}, 2 \mathrm{H}), 6.85(\mathrm{~m}, 2 \mathrm{H}), 6.47(\mathrm{~s}, 1 \mathrm{H})$, $3.80(\mathrm{~s}, 3 \mathrm{H}), 3.51(\mathrm{~s}, 3 \mathrm{H}) ;{ }^{13} \mathrm{C} \mathrm{NMR}\left(\mathrm{CDCl}_{3}, 100 \mathrm{MHz}\right) \delta 164.2,163.7,161.5,160.7,143.8,130.2,128.7,128.4$, 127.5, 126.7, 113.8, 97.3, 55.2, 38.3; Anal. Calcd for $\mathrm{C}_{18} \mathrm{H}_{16} \mathrm{ClN}_{3} \mathrm{O}$ : C, 66.36; H, 4.95; N, 12.90. Found: C, $66.13 ; \mathrm{H}, 5.01 ; \mathrm{N}, 12.73$.<smiles>COc1ccc(-c2cc(Cl)nc(N(C)c3ccccc3)n2)cc1</smiles>

12d

[4-Chloro-6-(4-methoxyphenyl)-pyrimidin-2-yl]methylphenylamine (12d). white solid: $\mathrm{mp} 110-111{ }^{\circ} \mathrm{C}$; ${ }^{1} \mathrm{H}$ NMR $\left(\mathrm{CDCl}_{3}, 400 \mathrm{MHz}\right) \delta 7.91(\mathrm{~d}, J=8.8 \mathrm{~Hz}, 2 \mathrm{H}), 7.42(\mathrm{~m}, 4 \mathrm{H}), 7.26(\mathrm{~m}, 1 \mathrm{H}), 7.01(\mathrm{~s}, 1 \mathrm{H}), 6.93(\mathrm{~d}, J=8.8$ $\mathrm{Hz}, 2 \mathrm{H}), 3.85$ (s, 3H), $3.63(\mathrm{~s}, 3 \mathrm{H}) ;{ }^{13} \mathrm{C} \mathrm{NMR}\left(\mathrm{CDCl}_{3}, 100 \mathrm{MHz}\right) \delta 165.0,162.0,161.5,161.4,145.0,128.8$, 128.71, 128.68, 126.3, 125.5, 114.0, 105.0, 55.3, 38.6; Anal. Calcd for $\mathrm{C}_{18} \mathrm{H}_{16} \mathrm{ClN}_{3} \mathrm{O}$ : C, 66.36; H, 4.95; N, 12.90. Found: C, 66.15; H, 4.94; N, 12.73 . 
<smiles>CC1CN(C(=O)OCc2ccccc2)CCN1c1cc(-c2ccccc2)nc(Cl)n1</smiles>

(R)-4-(2-Chloro-6-phenylpyrimidin-4-yl)-3-methyl-piperazine-1-carboxylic acid benzyl ester (14). colorless oil: ${ }^{1} \mathrm{H}$ NMR $\left(\mathrm{CDCl}_{3}, 400 \mathrm{MHz}\right) \delta 7.93(\mathrm{~m}, 2 \mathrm{H}), 7.44(\mathrm{~m}, 3 \mathrm{H}), 7.37(\mathrm{~m}, 5 \mathrm{H}), 6.71(\mathrm{~s}, 1 \mathrm{H}), 5.21(\mathrm{~d}, J=$ 12.3, Hz, 1H), $5.17(\mathrm{~d}, J=12.3 \mathrm{~Hz}, 1 \mathrm{H}), 4.55$ (br, 1H), 4.19 (br, 2H), 4.00 (br, 1H), 3.24 (m, 2H), 3.07 (br, 1H), $1.25(\mathrm{br}, 3 \mathrm{H}) ;{ }^{13} \mathrm{C} \mathrm{NMR}\left(\mathrm{CDCl}_{3}, 100 \mathrm{MHz}\right) \delta 165.6,163.1,160.7,155.4,136.4,136.2,130.5,128.5,128.3$, 128.0, 127.7, 126.9, 96.7, 67.2, 47.6, 47.1, 43.0, 38.5, 14.3; $[\alpha]^{23}{ }_{\mathrm{D}}-75.4\left(c 1.10, \mathrm{CH}_{2} \mathrm{Cl}_{2}\right)$; Anal. Calcd for $\mathrm{C}_{23} \mathrm{H}_{23} \mathrm{ClN}_{4} \mathrm{O}_{2}$ : C, 65.32; H, 5.48; N, 13.25. Found: C, 65.07; H, 5.67; N, 13.03.<smiles>CC1CN(C(=O)OCc2ccccc2)CCN1c1nc(Cl)cc(-c2ccccc2)n1</smiles>

15

(R)-4-(4-Chloro-6-phenylpyrimidin-2-yl)-3-methyl-piperazine-1-carboxylic acid benzyl ester (15). colorless oil: ${ }^{1} \mathrm{H} \mathrm{NMR}\left(\mathrm{CDCl}_{3}, 400 \mathrm{MHz}\right) \delta 8.01(\mathrm{~m}, 2 \mathrm{H}), 7.47(\mathrm{~m}, 3 \mathrm{H}), 7.39(\mathrm{~m}, 5 \mathrm{H}), 6.99(\mathrm{~s}, 1 \mathrm{H}), 5.24(\mathrm{~d}, J=$ 12.3, Hz, 1H), 5.19 (d, J = 12.3 Hz, 1H), 5.07 (br, 1H), 4.64 (br, 1H), 4.29-4.01 (br, 2H), 3.34-3.21 (m, 2H), $3.05(\mathrm{br}, 1 \mathrm{H}), 1.26(\mathrm{br}, 3 \mathrm{H}) ;{ }^{13} \mathrm{C} \mathrm{NMR}\left(\mathrm{CDCl}_{3}, 100 \mathrm{MHz}\right) \delta 165.9,161.8,161.0,155.7,136.5,136.4,130.9$, 128.7, 128.4, 128.0, 127.8, 127.0, 105.2, 67.2, 48.1, 47.6, 43.6, 38.3, 14.3; $[\alpha]^{23}{ }_{\mathrm{D}}-80.7\left(c 0.91, \mathrm{CH}_{2} \mathrm{Cl}_{2}\right) ; \mathrm{Anal}$. Calcd for $\mathrm{C}_{23} \mathrm{H}_{23} \mathrm{ClN}_{4} \mathrm{O}_{2}$ : C, 65.32; H, 5.48; N, 13.25. Found: C, 65.30; H, 5.53; N, 13.09. 
VII. Chiral HPLC traces of $( \pm)-13,(-)-13,( \pm)-14$ and (-)-14:

13: HPLC Method: Chiralpak AD-H (250 x $4.6 \mathrm{~mm}), 5 \%$ (MeOH: EtOH 1:1)/95\% Heptane with 0.1\% DEA, $1.5 \mathrm{ml} / \mathrm{min}, 265 \mathrm{~nm}, 25 \mathrm{~min}$ :
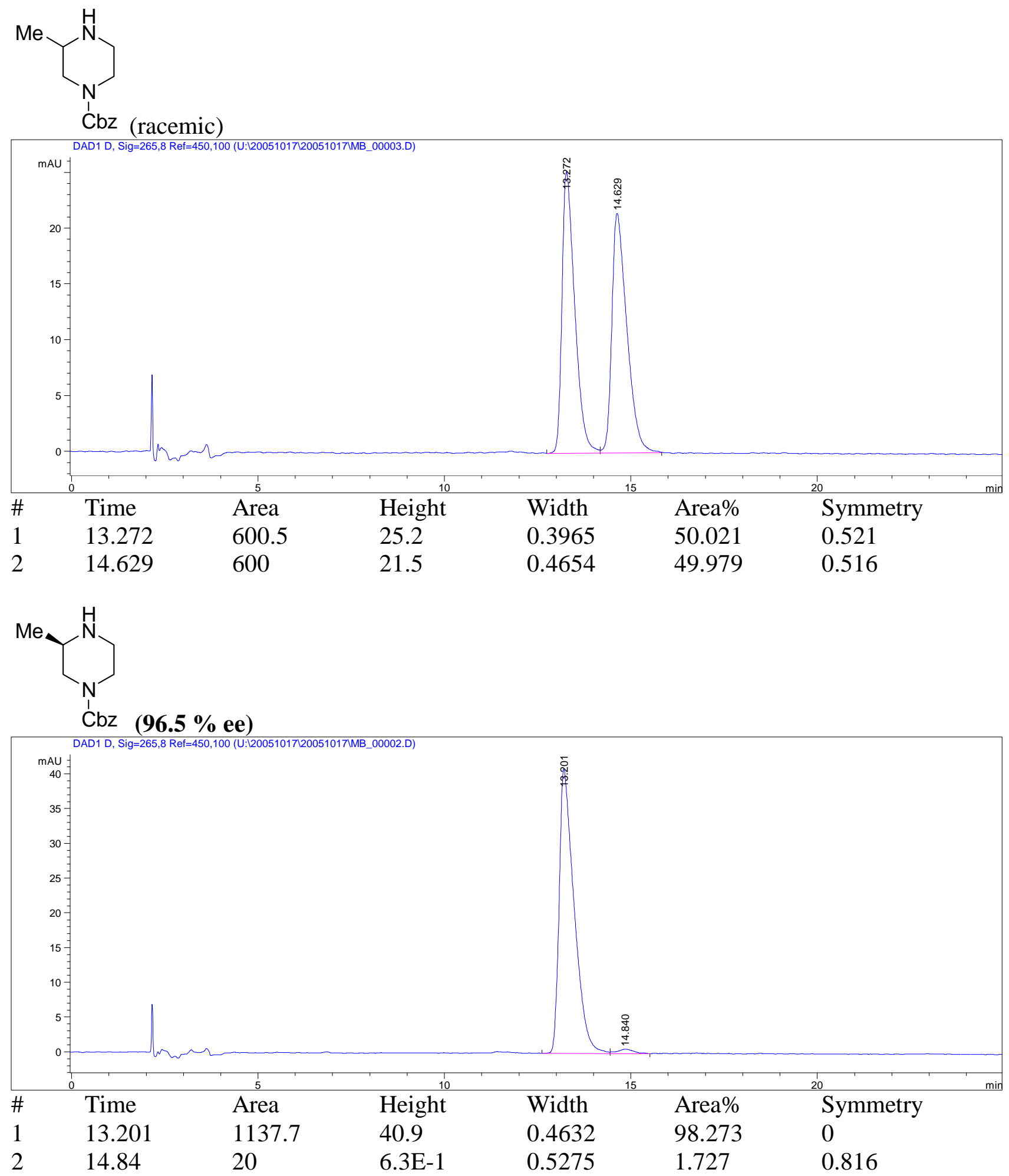
14: HPLC Method: Chiralpak AD-H (250 x $4.6 \mathrm{~mm}$ ), isocratic 8\% (MeOH:EtOH 1/1)/92\% Heptane with $0.1 \%$ DEA, $1.5 \mathrm{ml} / \mathrm{min}, 260 \mathrm{~nm}, 25 \mathrm{~min}$ :
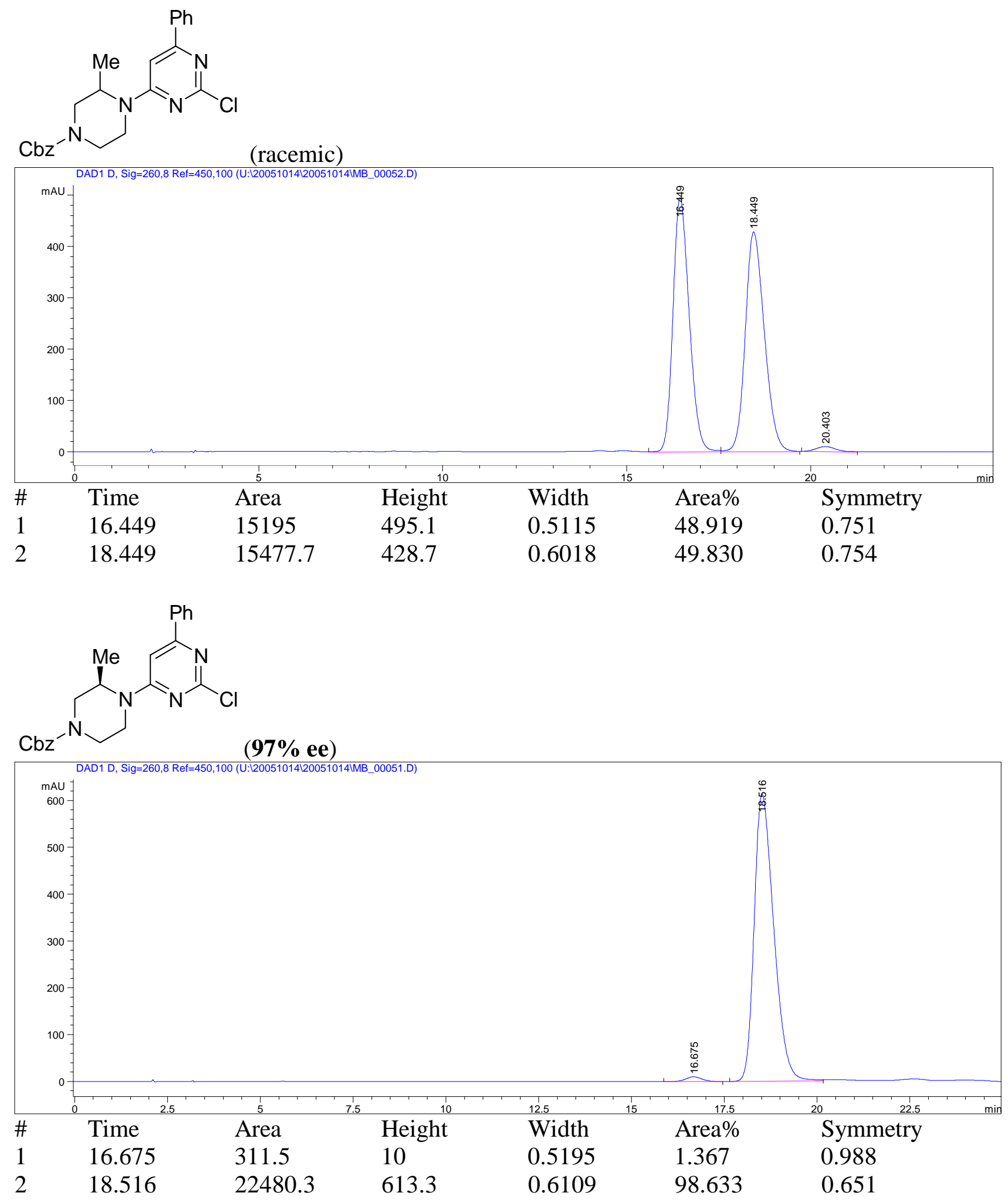


\section{X-ray crystallographic determination information for compound 6}<smiles>P=[PH](Cl)([PbH2])([PbH2])c1cc(Cl)nc(Cl)n1</smiles>

A needle of $\mathrm{C}_{40} \mathrm{H}_{31} \mathrm{Cl}_{3} \mathrm{~N}_{2} \mathrm{P}_{2} \mathrm{Pd}$, approximate dimensions $0.38 \mathrm{~mm}$ x $0.14 \mathrm{~mm}$ x $0.08 \mathrm{~mm}$ was used for the X-ray crystallographic analysis. The X-ray intensity data were measured at 298(2) K on a Bruker SMART APEX CCD area detector system equipped with a graphite monochromator and a Mo K $\alpha$ fine-focus sealed tube $(\lambda=$ $0.71073 \AA$ A) operated at $1.5 \mathrm{~kW}$ power $(50 \mathrm{kV}, 30 \mathrm{~mA})$.

A full quadrant of data (total of 2400 frames) was collected with an exposure time of 2 sec/frame. The frames were integrated with the Bruker SAINT software package using a narrow frame integration algorithm. The integration of the data using a monoclinic unit cell yielded a total of 78407 reflections to a maximum $\theta$ angle of $26.46^{\circ}$, of which 15318 were independent. Data were corrected for absorption effects using the multi-scan technique (SADABS).

The structure was solved and refined using the Bruker SHELXTL software package, using the space group $\mathrm{P} 2{ }_{1} / \mathrm{c}$, with $\mathrm{Z}=8$ for the formula unit, $\mathrm{C}_{40} \mathrm{H}_{31} \mathrm{Cl}_{3} \mathrm{~N}_{2} \mathrm{P}_{2} \mathrm{Pd}$. The final anisotropic full-matrix least-squares refinement on $\mathrm{F}^{2}$ with 865 variables converged at $\mathrm{R} 1=5.52 \%$, for the observed data and $\mathrm{wR} 2=12.53 \%$ for all data. The goodness-of-fit was 1.004. The largest peak on the final difference electron density map was $0.575 \mathrm{e}^{-}$ $/ \AA^{3}$ and the largest hole was $-0.435 \mathrm{e}^{-} / \AA^{3}$. On the basis of the final model, the calculated density was 1.450 $\mathrm{g} / \mathrm{cm}^{3}$. See the following tables for information pertaining to the final specifications of the solved structure. 


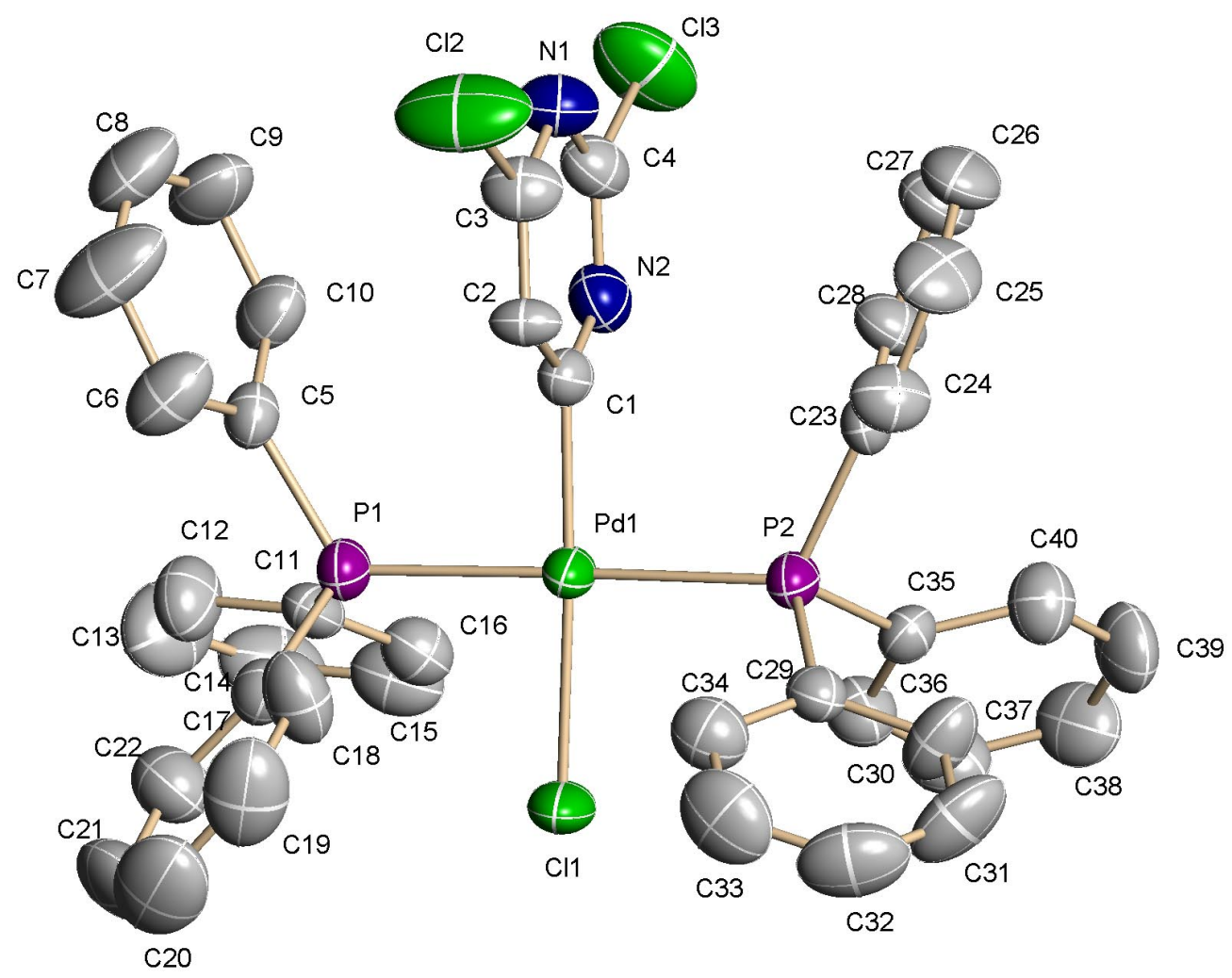

ORTEP view of molecule $\mathrm{C}_{40} \mathrm{H}_{31} \mathrm{Cl}_{3} \mathrm{~N}_{2} \mathrm{P}_{2} \mathrm{Pd}$. Anisotropic atomic displacement ellipsoids for the non-hydrogen atoms are shown at the 50\% probability level. Hydrogen atoms have been excluded for clarity. 
Table 1. Crystal data and structure refinement for compound 6.

Identification code

Empirical formula

Formula weight

Temperature

Wavelength

Crystal system

Space group

Unit cell dimensions

Volume

Z

Density (calculated)

Absorption coefficient

$\mathrm{F}(000)$

Crystal size

Theta range for data collection

Index ranges

Reflections collected

Independent reflections

Completeness to theta $=26.46^{\circ}$

Absorption correction

Refinement method

Data / restraints / parameters

Goodness-of-fit on $\mathrm{F}^{2}$

Final $\mathrm{R}$ indices [I $>2 \operatorname{sigma}(\mathrm{I})]$

$\mathrm{R}$ indices (all data)

Largest diff. peak and hole compound 6

C40 H31 Cl3 N2 P2 Pd

814.36

298(2) K

$0.71073 \AA$

Monoclinic

P2(1)/c

$\begin{array}{ll}\mathrm{a}=26.119(3) \AA & \alpha=90^{\circ} . \\ \mathrm{b}=11.3717(13) \AA & \beta=100.205(2)^{\circ} . \\ \mathrm{c}=25.521(3) \AA & \gamma=90^{\circ} .\end{array}$

7460.4(14) $\AA^{3}$

8

$1.450 \mathrm{Mg} / \mathrm{m}^{3}$

$0.829 \mathrm{~mm}^{-1}$

3296

$0.38 \times 0.14 \times 0.08 \mathrm{~mm}^{3}$

0.79 to $26.46^{\circ}$.

$-32<=\mathrm{h}<=32,-14<=\mathrm{k}<=14,-31<=\mathrm{l}<=31$

78407

15318 [R(int $)=0.1032]$

$99.5 \%$

None

Full-matrix least-squares on $\mathrm{F}^{2}$

15318 / 0 / 865

1.005

$\mathrm{R} 1=0.0552, \mathrm{wR} 2=0.1054$

$\mathrm{R} 1=0.1119, \mathrm{wR} 2=0.1252$

0.575 and -0.435 e. $\AA^{-3}$

Table 2. Atomic coordinates ( $\left.\mathrm{x} 10^{4}\right)$ and equivalent isotropic displacement parameters $\left(\AA^{2} \times 10^{3}\right)$ for compound 6 . U(eq) is defined as one third of the trace of the orthogonalized $U^{i j}$ tensor.

\begin{tabular}{lrrrr}
\hline & $\mathrm{x}$ & $\mathrm{y}$ & $\mathrm{z}$ & $\mathrm{U}(\mathrm{eq})$ \\
\hline $\mathrm{C}(1)$ & & & & \\
$\mathrm{C}(2)$ & $1229(2)$ & $7212(4)$ & $1079(2)$ & $39(1)$ \\
$\mathrm{C}(3)$ & $1688(2)$ & $6679(4)$ & $1268(2)$ & $38(1)$ \\
$\mathrm{C}(4)$ & $1838(2)$ & $5808(4)$ & $974(2)$ & $53(1)$ \\
$\mathrm{C}(5)$ & $1129(2)$ & $5962(4)$ & $365(2)$ & $49(1)$ \\
$\mathrm{C}(6)$ & $1943(2)$ & $9216(4)$ & $673(2)$ & $43(1)$ \\
$\mathrm{C}(7)$ & $2467(2)$ & $9140(6)$ & $868(2)$ & $75(2)$ \\
& $2794(2)$ & $8574(7)$ & $577(3)$ & $106(3)$
\end{tabular}




\begin{tabular}{|c|c|c|c|c|}
\hline $\mathrm{C}(8)$ & $2603(3)$ & $8074(6)$ & 101(3) & $88(2)$ \\
\hline $\mathrm{C}(9)$ & $2092(2)$ & $8163(6)$ & $-104(2)$ & $80(2)$ \\
\hline $\mathrm{C}(10)$ & $1760(2)$ & $8730(5)$ & $184(2)$ & $60(2)$ \\
\hline $\mathrm{C}(11)$ & $1092(2)$ & $10811(4)$ & $590(2)$ & $45(1)$ \\
\hline$C(12)$ & $1313(2)$ & $11543(5)$ & $258(2)$ & $67(2)$ \\
\hline $\mathrm{C}(13)$ & $1010(3)$ & $12264(6)$ & $-100(3)$ & $90(2)$ \\
\hline $\mathrm{C}(14)$ & 483(3) & $12232(5)$ & $-140(2)$ & $83(2)$ \\
\hline$C(15)$ & $251(2)$ & $11515(5)$ & $177(2)$ & $71(2)$ \\
\hline$C(16)$ & $559(2)$ & $10810(4)$ & $548(2)$ & $54(1)$ \\
\hline $\mathrm{C}(17)$ & $1873(2)$ & $10703(4)$ & $1582(2)$ & $47(1)$ \\
\hline $\mathrm{C}(18)$ & $2100(2)$ & $10131(5)$ & $2038(2)$ & $73(2)$ \\
\hline C(19) & $2376(3)$ & $10731(8)$ & $2467(2)$ & $94(2)$ \\
\hline$C(20)$ & $2404(3)$ & 11923(9) & $2440(3)$ & $106(3)$ \\
\hline $\mathrm{C}(21)$ & 2193(3) & $12502(6)$ & 1996(3) & $95(2)$ \\
\hline $\mathrm{C}(22)$ & $1926(2)$ & $11911(5)$ & $1562(2)$ & $69(2)$ \\
\hline $\mathrm{C}(23)$ & $578(2)$ & $5710(4)$ & 1797(2) & $38(1)$ \\
\hline $\mathrm{C}(24)$ & $873(2)$ & $4985(4)$ & $2168(2)$ & $55(1)$ \\
\hline $\mathrm{C}(25)$ & $946(2)$ & $3823(5)$ & $2048(2)$ & $72(2)$ \\
\hline$C(26)$ & $735(2)$ & $3368(5)$ & $1560(3)$ & $69(2)$ \\
\hline $\mathrm{C}(27)$ & $447(2)$ & $4075(4)$ & $1188(2)$ & $60(1)$ \\
\hline $\mathrm{C}(28)$ & $368(2)$ & $5230(4)$ & $1306(2)$ & $51(1)$ \\
\hline C(29) & $682(2)$ & $7446(4)$ & $2625(2)$ & $40(1)$ \\
\hline$C(30)$ & $379(2)$ & $7000(5)$ & $2965(2)$ & $65(2)$ \\
\hline $\mathrm{C}(31)$ & $533(3)$ & $7085(6)$ & $3506(2)$ & $82(2)$ \\
\hline$C(32)$ & $978(3)$ & $7645(6)$ & $3718(2)$ & $86(2)$ \\
\hline $\mathrm{C}(33)$ & 1291(2) & $8107(6)$ & $3382(3)$ & $84(2)$ \\
\hline $\mathrm{C}(34)$ & $1138(2)$ & $8000(5)$ & $2838(2)$ & $60(1)$ \\
\hline$C(35)$ & $-208(2)$ & $7473(4)$ & $1750(2)$ & $40(1)$ \\
\hline$C(36)$ & $-410(2)$ & $8541(4)$ & $1563(2)$ & $53(1)$ \\
\hline $\mathrm{C}(37)$ & $-944(2)$ & $8729(5)$ & $1455(2)$ & $69(2)$ \\
\hline $\mathrm{C}(38)$ & $-1272(2)$ & $7832(6)$ & $1534(2)$ & $73(2)$ \\
\hline C(39) & $-1078(2)$ & $6763(6)$ & $1720(2)$ & $71(2)$ \\
\hline$C(40)$ & $-549(2)$ & $6585(5)$ & $1828(2)$ & $58(1)$ \\
\hline $\mathrm{C}(41)$ & $3786(2)$ & $1043(4)$ & $4000(2)$ & $41(1)$ \\
\hline$C(42)$ & $3925(2)$ & $878(4)$ & $4527(2)$ & $41(1)$ \\
\hline$C(43)$ & $3728(2)$ & $-41(5)$ & $4744(2)$ & $61(2)$ \\
\hline $\mathrm{C}(44)$ & $3289(2)$ & $-639(4)$ & $3971(2)$ & $56(1)$ \\
\hline$C(45)$ & $4521(2)$ & $-345(4)$ & $3385(2)$ & $54(1)$ \\
\hline$C(46)$ & $4733(2)$ & $-681(5)$ & $3904(2)$ & $68(2)$ \\
\hline$C(47)$ & $4689(3)$ & $-1845(6)$ & $4063(3)$ & $93(2)$ \\
\hline $\mathrm{C}(48)$ & $4436(3)$ & $-2633(6)$ & $3710(4)$ & $104(3)$ \\
\hline C(49) & $4236(3)$ & $-2323(6)$ & $3209(3)$ & $101(2)$ \\
\hline $\mathrm{C}(50)$ & $4275(2)$ & $-1171(5)$ & $3038(3)$ & $79(2)$ \\
\hline $\mathrm{C}(51)$ & $5255(2)$ & $1433(5)$ & $3263(2)$ & $49(1)$ \\
\hline $\mathrm{C}(52)$ & $5577(2)$ & 499(5) & $3182(2)$ & $72(2)$ \\
\hline $\mathrm{C}(53)$ & $6096(2)$ & $672(6)$ & $3184(2)$ & $83(2)$ \\
\hline
\end{tabular}




\begin{tabular}{|c|c|c|c|c|}
\hline$C(54)$ & $6309(2)$ & $1774(7)$ & $3284(3)$ & $85(2)$ \\
\hline$C(55)$ & $5995(2)$ & $2708(6)$ & $3362(2)$ & $81(2)$ \\
\hline$C(56)$ & $5469(2)$ & $2527(5)$ & $3355(2)$ & $64(2)$ \\
\hline$C(57)$ & $4293(2)$ & $1329(5)$ & $2521(2)$ & $57(1)$ \\
\hline $\mathrm{C}(58)$ & $4578(2)$ & $1155(8)$ & $2137(2)$ & $113(3)$ \\
\hline$C(59)$ & $4364(3)$ & $1230(10)$ & $1608(3)$ & $169(5)$ \\
\hline$C(60)$ & $3860(4)$ & $1520(9)$ & $1463(3)$ & $149(4)$ \\
\hline $\mathrm{C}(61)$ & $3562(3)$ & $1643(10)$ & $1836(3)$ & $163(4)$ \\
\hline$C(62)$ & $3782(3)$ & $1577(8)$ & $2363(3)$ & $123(3)$ \\
\hline$C(63)$ & $3821(2)$ & $4599(4)$ & $4532(2)$ & $46(1)$ \\
\hline$C(64)$ & $3705(2)$ & $5754(5)$ & $4563(2)$ & $60(1)$ \\
\hline$C(65)$ & $3990(2)$ & $6451(5)$ & $4959(2)$ & $74(2)$ \\
\hline$C(66)$ & $4378(2)$ & $6014(6)$ & $5312(2)$ & $72(2)$ \\
\hline$C(67)$ & $4502(2)$ & $4847(6)$ & $5285(2)$ & $81(2)$ \\
\hline $\mathrm{C}(68)$ & $4228(2)$ & $4152(5)$ & $4892(2)$ & $74(2)$ \\
\hline$C(69)$ & $3028(2)$ & $2818(4)$ & $4403(2)$ & $44(1)$ \\
\hline $\mathrm{C}(70)$ & $2675(2)$ & $2049(4)$ & $4130(2)$ & $57(1)$ \\
\hline $\mathrm{C}(71)$ & $2350(2)$ & 1391(5) & $4392(3)$ & $73(2)$ \\
\hline $\mathrm{C}(72)$ & $2378(3)$ & $1526(6)$ & 4927(3) & $92(2)$ \\
\hline $\mathrm{C}(73)$ & $2720(3)$ & $2309(7)$ & $5206(3)$ & $103(2)$ \\
\hline $\mathrm{C}(74)$ & $3039(2)$ & $2954(5)$ & $4944(2)$ & $72(2)$ \\
\hline$C(75)$ & $3012(2)$ & $4477(4)$ & $3580(2)$ & $46(1)$ \\
\hline$C(76)$ & $3102(2)$ & $4723(5)$ & $3076(2)$ & $64(2)$ \\
\hline $\mathrm{C}(77)$ & $2750(3)$ & $5379(5)$ & $2728(2)$ & $81(2)$ \\
\hline $\mathrm{C}(78)$ & $2309(2)$ & $5792(5)$ & $2877(3)$ & $80(2)$ \\
\hline $\mathrm{C}(79)$ & $2206(2)$ & $5546(5)$ & $3374(3)$ & $73(2)$ \\
\hline $\mathrm{C}(80)$ & $2561(2)$ & 4903(4) & $3728(2)$ & $58(1)$ \\
\hline $\mathrm{Cl}(1)$ & 724(1) & $10193(1)$ & $1929(1)$ & $54(1)$ \\
\hline $\mathrm{Cl}(2)$ & $2439(1)$ & $5142(2)$ & $1179(1)$ & 111(1) \\
\hline $\mathrm{Cl}(3)$ & 747(1) & $5477(1)$ & $-216(1)$ & $92(1)$ \\
\hline $\mathrm{Cl}(4)$ & $4394(1)$ & $4203(1)$ & $3356(1)$ & $74(1)$ \\
\hline $\mathrm{Cl}(5)$ & $3876(1)$ & $-265(2)$ & $5424(1)$ & $115(1)$ \\
\hline $\mathrm{Cl}(6)$ & $2879(1)$ & $-1644(2)$ & 3601(1) & $106(1)$ \\
\hline $\mathrm{N}(1)$ & $1570(2)$ & $5395(4)$ & $521(2)$ & $58(1)$ \\
\hline $\mathrm{N}(2)$ & $937(2)$ & $6862(3)$ & $609(2)$ & $50(1)$ \\
\hline $\mathrm{N}(3)$ & $3406(2)$ & $-838(4)$ & $4488(2)$ & $65(1)$ \\
\hline $\mathrm{N}(4)$ & $3455(2)$ & 277(4) & $3698(2)$ & $58(1)$ \\
\hline $\mathrm{P}(1)$ & $1485(1)$ & $9829(1)$ & $1060(1)$ & $39(1)$ \\
\hline $\mathrm{P}(2)$ & 497(1) & $7274(1)$ & $1906(1)$ & $36(1)$ \\
\hline $\mathrm{P}(3)$ & $4558(1)$ & $1208(1)$ & $3222(1)$ & $46(1)$ \\
\hline $\mathrm{P}(4)$ & $3467(1)$ & $3596(1)$ & $4043(1)$ & 41(1) \\
\hline $\operatorname{Pd}(1)$ & 980(1) & $8547(1)$ & $1465(1)$ & $36(1)$ \\
\hline $\operatorname{Pd}(2)$ & $4053(1)$ & $2446(1)$ & $3670(1)$ & 41(1) \\
\hline
\end{tabular}


Table 3. Bond lengths $[\AA]$ and angles $\left[{ }^{\circ}\right]$ for compound $\mathbf{6}$.

\begin{tabular}{ll}
\hline $\mathrm{C}(1)-\mathrm{C}(2)$ & $1.355(6)$ \\
$\mathrm{C}(1)-\mathrm{N}(2)$ & $1.360(5)$ \\
$\mathrm{C}(1)-\mathrm{Pd}(1)$ & $1.980(4)$ \\
$\mathrm{C}(2)-\mathrm{C}(3)$ & $1.341(6)$ \\
$\mathrm{C}(2)-\mathrm{H}(2)$ & 0.9300 \\
$\mathrm{C}(3)-\mathrm{N}(1)$ & $1.327(6)$ \\
$\mathrm{C}(3)-\mathrm{Cl}(2)$ & $1.738(5)$ \\
$\mathrm{C}(4)-\mathrm{N}(1)$ & $1.318(6)$ \\
$\mathrm{C}(4)-\mathrm{N}(2)$ & $1.341(6)$ \\
$\mathrm{C}(4)-\mathrm{Cl}(3)$ & $1.724(5)$ \\
$\mathrm{C}(5)-\mathrm{C}(10)$ & $1.370(6)$ \\
$\mathrm{C}(5)-\mathrm{C}(6)$ & $1.374(6)$ \\
$\mathrm{C}(5)-\mathrm{P}(1)$ & $1.820(5)$ \\
$\mathrm{C}(6)-\mathrm{C}(7)$ & $1.387(8)$ \\
$\mathrm{C}(6)-\mathrm{H}(6)$ & 0.9300 \\
$\mathrm{C}(7)-\mathrm{C}(8)$ & $1.353(8)$ \\
$\mathrm{C}(7)-\mathrm{H}(7)$ & 0.9300 \\
$\mathrm{C}(8)-\mathrm{C}(9)$ & $1.348(8)$ \\
$\mathrm{C}(8)-\mathrm{H}(8)$ & 0.9300 \\
$\mathrm{C}(9)-\mathrm{C}(10)$ & $1.390(7)$ \\
$\mathrm{C}(9)-\mathrm{H}(9)$ & 0.9300 \\
$\mathrm{C}(10)-\mathrm{H}(10)$ & 0.9300 \\
$\mathrm{C}(11)-\mathrm{C}(16)$ & $1.375(6)$ \\
$\mathrm{C}(11)-\mathrm{C}(12)$ & $1.385(6)$ \\
$\mathrm{C}(11)-\mathrm{P}(1)$ & $1.818(5)$ \\
$\mathrm{C}(12)-\mathrm{C}(13)$ & $1.371(7)$ \\
$\mathrm{C}(12)-\mathrm{H}(12)$ & 0.9300 \\
$\mathrm{C}(13)-\mathrm{C}(14)$ & $1.362(9)$ \\
$\mathrm{C}(13)-\mathrm{H}(13)$ & 0.9300 \\
$\mathrm{C}(14)-\mathrm{C}(15)$ & $1.367(8)$ \\
$\mathrm{C}(14)-\mathrm{H}(14)$ & 0.9300 \\
$\mathrm{C}(15)-\mathrm{C}(16)$ & $1.385(7)$ \\
$\mathrm{C}(15)-\mathrm{H}(15)$ & 0.9300 \\
$\mathrm{C}(16)-\mathrm{H}(16)$ & 0.9300 \\
$\mathrm{C}(17)-\mathrm{C}(18)$ & $1.372(7)$ \\
$\mathrm{C}(17)-\mathrm{C}(22)$ & $1.382(7)$ \\
$\mathrm{C}(17)-\mathrm{P}(1)$ & $1.821(5)$ \\
$\mathrm{C}(18)-\mathrm{C}(19)$ & $1.381(8)$ \\
$\mathrm{C}(18)-\mathrm{H}(18)$ & 0.9300 \\
$\mathrm{C}(19)-\mathrm{C}(20)$ & $1.360(9)$ \\
$\mathrm{C}(19)-\mathrm{H}(19)$ & 0.9300 \\
$\mathrm{C}(20)-\mathrm{C}(21)$ & $1.341(9)$ \\
$\mathrm{C}(20)-\mathrm{H}(20)$ & 0.9300 \\
$\mathrm{C}(21)-\mathrm{C}(22)$ & $1.377(8)$ \\
&
\end{tabular}




\begin{tabular}{|c|c|}
\hline $\mathrm{C}(21)-\mathrm{H}(21)$ & 0.9300 \\
\hline $\mathrm{C}(22)-\mathrm{H}(22)$ & 0.9300 \\
\hline $\mathrm{C}(23)-\mathrm{C}(24)$ & $1.382(6)$ \\
\hline $\mathrm{C}(23)-\mathrm{C}(28)$ & $1.387(6)$ \\
\hline $\mathrm{C}(23)-\mathrm{P}(2)$ & $1.818(4)$ \\
\hline $\mathrm{C}(24)-\mathrm{C}(25)$ & $1.377(7)$ \\
\hline $\mathrm{C}(24)-\mathrm{H}(24)$ & 0.9300 \\
\hline$C(25)-C(26)$ & $1.371(7)$ \\
\hline $\mathrm{C}(25)-\mathrm{H}(25)$ & 0.9300 \\
\hline$C(26)-C(27)$ & $1.363(7)$ \\
\hline $\mathrm{C}(26)-\mathrm{H}(26)$ & 0.9300 \\
\hline $\mathrm{C}(27)-\mathrm{C}(28)$ & $1.373(6)$ \\
\hline $\mathrm{C}(27)-\mathrm{H}(27)$ & 0.9300 \\
\hline $\mathrm{C}(28)-\mathrm{H}(28)$ & 0.9300 \\
\hline C(29)-C(30) & $1.370(6)$ \\
\hline C(29)-C(34) & $1.373(6)$ \\
\hline $\mathrm{C}(29)-\mathrm{P}(2)$ & $1.824(5)$ \\
\hline $\mathrm{C}(30)-\mathrm{C}(31)$ & $1.371(7)$ \\
\hline $\mathrm{C}(30)-\mathrm{H}(30)$ & 0.9300 \\
\hline $\mathrm{C}(31)-\mathrm{C}(32)$ & $1.352(8)$ \\
\hline $\mathrm{C}(31)-\mathrm{H}(31)$ & 0.9300 \\
\hline $\mathrm{C}(32)-\mathrm{C}(33)$ & $1.388(8)$ \\
\hline $\mathrm{C}(32)-\mathrm{H}(32)$ & 0.9300 \\
\hline $\mathrm{C}(33)-\mathrm{C}(34)$ & $1.380(7)$ \\
\hline C(33)-H(33) & 0.9300 \\
\hline C(34)-H(34) & 0.9300 \\
\hline $\mathrm{C}(35)-\mathrm{C}(36)$ & $1.376(6)$ \\
\hline$C(35)-C(40)$ & $1.385(6)$ \\
\hline $\mathrm{C}(35)-\mathrm{P}(2)$ & $1.828(5)$ \\
\hline$C(36)-C(37)$ & $1.390(7)$ \\
\hline $\mathrm{C}(36)-\mathrm{H}(36)$ & 0.9300 \\
\hline $\mathrm{C}(37)-\mathrm{C}(38)$ & $1.369(7)$ \\
\hline $\mathrm{C}(37)-\mathrm{H}(37)$ & 0.9300 \\
\hline C(38)-C(39) & $1.370(7)$ \\
\hline $\mathrm{C}(38)-\mathrm{H}(38)$ & 0.9300 \\
\hline C(39)-C(40) & $1.375(7)$ \\
\hline C(39)-H(39) & 0.9300 \\
\hline $\mathrm{C}(40)-\mathrm{H}(40)$ & 0.9300 \\
\hline $\mathrm{C}(41)-\mathrm{C}(42)$ & $1.343(6)$ \\
\hline $\mathrm{C}(41)-\mathrm{N}(4)$ & $1.365(6)$ \\
\hline $\mathrm{C}(41)-\mathrm{Pd}(2)$ & $1.987(4)$ \\
\hline $\mathrm{C}(42)-\mathrm{C}(43)$ & $1.330(7)$ \\
\hline $\mathrm{C}(42)-\mathrm{H}(42)$ & 0.9300 \\
\hline $\mathrm{C}(43)-\mathrm{N}(3)$ & $1.327(6)$ \\
\hline $\mathrm{C}(43)-\mathrm{Cl}(5)$ & $1.728(5)$ \\
\hline C(44)-N(3) & $1.319(6)$ \\
\hline
\end{tabular}




\begin{tabular}{|c|c|}
\hline $\mathrm{C}(44)-\mathrm{N}(4)$ & $1.367(6)$ \\
\hline $\mathrm{C}(44)-\mathrm{Cl}(6)$ & $1.728(5)$ \\
\hline$C(45)-C(50)$ & $1.370(7)$ \\
\hline$C(45)-C(46)$ & $1.395(7)$ \\
\hline $\mathrm{C}(45)-\mathrm{P}(3)$ & $1.820(5)$ \\
\hline $\mathrm{C}(46)-\mathrm{C}(47)$ & $1.396(8)$ \\
\hline $\mathrm{C}(46)-\mathrm{H}(46)$ & 0.9300 \\
\hline $\mathrm{C}(47)-\mathrm{C}(48)$ & $1.356(9)$ \\
\hline $\mathrm{C}(47)-\mathrm{H}(47)$ & 0.9300 \\
\hline $\mathrm{C}(48)-\mathrm{C}(49)$ & $1.341(9)$ \\
\hline $\mathrm{C}(48)-\mathrm{H}(48)$ & 0.9300 \\
\hline $\mathrm{C}(49)-\mathrm{C}(50)$ & $1.390(8)$ \\
\hline C(49)-H(49) & 0.9300 \\
\hline $\mathrm{C}(50)-\mathrm{H}(50)$ & 0.9300 \\
\hline $\mathrm{C}(51)-\mathrm{C}(56)$ & $1.366(7)$ \\
\hline $\mathrm{C}(51)-\mathrm{C}(52)$ & $1.392(7)$ \\
\hline $\mathrm{C}(51)-\mathrm{P}(3)$ & $1.824(5)$ \\
\hline$C(52)-C(53)$ & $1.371(7)$ \\
\hline $\mathrm{C}(52)-\mathrm{H}(52)$ & 0.9300 \\
\hline $\mathrm{C}(53)-\mathrm{C}(54)$ & $1.376(8)$ \\
\hline $\mathrm{C}(53)-\mathrm{H}(53)$ & 0.9300 \\
\hline $\mathrm{C}(54)-\mathrm{C}(55)$ & $1.378(8)$ \\
\hline $\mathrm{C}(54)-\mathrm{H}(54)$ & 0.9300 \\
\hline$C(55)-C(56)$ & $1.386(7)$ \\
\hline $\mathrm{C}(55)-\mathrm{H}(55)$ & 0.9300 \\
\hline $\mathrm{C}(56)-\mathrm{H}(56)$ & 0.9300 \\
\hline $\mathrm{C}(57)-\mathrm{C}(58)$ & $1.345(7)$ \\
\hline$C(57)-C(62)$ & $1.353(7)$ \\
\hline $\mathrm{C}(57)-\mathrm{P}(3)$ & $1.806(5)$ \\
\hline $\mathrm{C}(58)-\mathrm{C}(59)$ & $1.371(8)$ \\
\hline $\mathrm{C}(58)-\mathrm{H}(58)$ & 0.9300 \\
\hline $\mathrm{C}(59)-\mathrm{C}(60)$ & $1.343(10)$ \\
\hline $\mathrm{C}(59)-\mathrm{H}(59)$ & 0.9300 \\
\hline$C(60)-C(61)$ & $1.343(9)$ \\
\hline $\mathrm{C}(60)-\mathrm{H}(60)$ & 0.9300 \\
\hline $\mathrm{C}(61)-\mathrm{C}(62)$ & $1.366(9)$ \\
\hline $\mathrm{C}(61)-\mathrm{H}(61)$ & 0.9300 \\
\hline $\mathrm{C}(62)-\mathrm{H}(62)$ & 0.9300 \\
\hline$C(63)-C(64)$ & $1.355(6)$ \\
\hline$C(63)-C(68)$ & $1.374(7)$ \\
\hline $\mathrm{C}(63)-\mathrm{P}(4)$ & $1.818(5)$ \\
\hline$C(64)-C(65)$ & $1.392(7)$ \\
\hline C(64)-H(64) & 0.9300 \\
\hline$C(65)-C(66)$ & $1.329(8)$ \\
\hline $\mathrm{C}(65)-\mathrm{H}(65)$ & 0.9300 \\
\hline $\mathrm{C}(66)-\mathrm{C}(67)$ & $1.369(8)$ \\
\hline
\end{tabular}




\begin{tabular}{|c|c|}
\hline $\mathrm{C}(66)-\mathrm{H}(66)$ & 0.9300 \\
\hline $\mathrm{C}(67)-\mathrm{C}(68)$ & $1.374(7)$ \\
\hline $\mathrm{C}(67)-\mathrm{H}(67)$ & 0.9300 \\
\hline $\mathrm{C}(68)-\mathrm{H}(68)$ & 0.9300 \\
\hline $\mathrm{C}(69)-\mathrm{C}(70)$ & $1.369(6)$ \\
\hline $\mathrm{C}(69)-\mathrm{C}(74)$ & $1.384(7)$ \\
\hline $\mathrm{C}(69)-\mathrm{P}(4)$ & $1.820(5)$ \\
\hline $\mathrm{C}(70)-\mathrm{C}(71)$ & $1.388(7)$ \\
\hline $\mathrm{C}(70)-\mathrm{H}(70)$ & 0.9300 \\
\hline $\mathrm{C}(71)-\mathrm{C}(72)$ & $1.363(8)$ \\
\hline $\mathrm{C}(71)-\mathrm{H}(71)$ & 0.9300 \\
\hline $\mathrm{C}(72)-\mathrm{C}(73)$ & $1.369(9)$ \\
\hline $\mathrm{C}(72)-\mathrm{H}(72)$ & 0.9300 \\
\hline $\mathrm{C}(73)-\mathrm{C}(74)$ & $1.370(8)$ \\
\hline $\mathrm{C}(73)-\mathrm{H}(73)$ & 0.9300 \\
\hline $\mathrm{C}(74)-\mathrm{H}(74)$ & 0.9300 \\
\hline$C(75)-C(76)$ & $1.377(6)$ \\
\hline$C(75)-C(80)$ & $1.387(6)$ \\
\hline $\mathrm{C}(75)-\mathrm{P}(4)$ & $1.820(5)$ \\
\hline $\mathrm{C}(76)-\mathrm{C}(77)$ & $1.379(7)$ \\
\hline $\mathrm{C}(76)-\mathrm{H}(76)$ & 0.9300 \\
\hline $\mathrm{C}(77)-\mathrm{C}(78)$ & $1.359(8)$ \\
\hline $\mathrm{C}(77)-\mathrm{H}(77)$ & 0.9300 \\
\hline $\mathrm{C}(78)-\mathrm{C}(79)$ & $1.371(8)$ \\
\hline $\mathrm{C}(78)-\mathrm{H}(78)$ & 0.9300 \\
\hline $\mathrm{C}(79)-\mathrm{C}(80)$ & $1.384(7)$ \\
\hline $\mathrm{C}(79)-\mathrm{H}(79)$ & 0.9300 \\
\hline $\mathrm{C}(80)-\mathrm{H}(80)$ & 0.9300 \\
\hline $\mathrm{Cl}(1)-\mathrm{Pd}(1)$ & $2.3739(11)$ \\
\hline $\mathrm{Cl}(4)-\mathrm{Pd}(2)$ & $2.3823(13)$ \\
\hline $\mathrm{P}(1)-\mathrm{Pd}(1)$ & $2.3264(12)$ \\
\hline $\mathrm{P}(2)-\mathrm{Pd}(1)$ & $2.3364(12)$ \\
\hline $\mathrm{P}(3)-\mathrm{Pd}(2)$ & $2.3572(12)$ \\
\hline $\mathrm{P}(4)-\mathrm{Pd}(2)$ & $2.3406(12)$ \\
\hline $\mathrm{C}(2)-\mathrm{C}(1)-\mathrm{N}(2)$ & $120.3(4)$ \\
\hline $\mathrm{C}(2)-\mathrm{C}(1)-\mathrm{Pd}(1)$ & $120.9(3)$ \\
\hline $\mathrm{N}(2)-\mathrm{C}(1)-\mathrm{Pd}(1)$ & $118.7(3)$ \\
\hline $\mathrm{C}(3)-\mathrm{C}(2)-\mathrm{C}(1)$ & $117.3(4)$ \\
\hline $\mathrm{C}(3)-\mathrm{C}(2)-\mathrm{H}(2)$ & 121.4 \\
\hline $\mathrm{C}(1)-\mathrm{C}(2)-\mathrm{H}(2)$ & 121.4 \\
\hline $\mathrm{N}(1)-\mathrm{C}(3)-\mathrm{C}(2)$ & $125.9(4)$ \\
\hline $\mathrm{N}(1)-\mathrm{C}(3)-\mathrm{Cl}(2)$ & $114.7(4)$ \\
\hline $\mathrm{C}(2)-\mathrm{C}(3)-\mathrm{Cl}(2)$ & $119.3(4)$ \\
\hline $\mathrm{N}(1)-\mathrm{C}(4)-\mathrm{N}(2)$ & $127.5(4)$ \\
\hline $\mathrm{N}(1)-\mathrm{C}(4)-\mathrm{Cl}(3)$ & $116.5(4)$ \\
\hline $\mathrm{N}(2)-\mathrm{C}(4)-\mathrm{Cl}(3)$ & $116.0(4)$ \\
\hline
\end{tabular}




$\begin{array}{ll}\mathrm{C}(10)-\mathrm{C}(5)-\mathrm{C}(6) & 118.0(5) \\ \mathrm{C}(10)-\mathrm{C}(5)-\mathrm{P}(1) & 119.4(4) \\ \mathrm{C}(6)-\mathrm{C}(5)-\mathrm{P}(1) & 122.4(4) \\ \mathrm{C}(5)-\mathrm{C}(6)-\mathrm{C}(7) & 120.2(5) \\ \mathrm{C}(5)-\mathrm{C}(6)-\mathrm{H}(6) & 119.9 \\ \mathrm{C}(7)-\mathrm{C}(6)-\mathrm{H}(6) & 119.9 \\ \mathrm{C}(8)-\mathrm{C}(7)-\mathrm{C}(6) & 120.8(6) \\ \mathrm{C}(8)-\mathrm{C}(7)-\mathrm{H}(7) & 119.6 \\ \mathrm{C}(6)-\mathrm{C}(7)-\mathrm{H}(7) & 119.6 \\ \mathrm{C}(9)-\mathrm{C}(8)-\mathrm{C}(7) & 119.9(6) \\ \mathrm{C}(9)-\mathrm{C}(8)-\mathrm{H}(8) & 120.0 \\ \mathrm{C}(7)-\mathrm{C}(8)-\mathrm{H}(8) & 120.0 \\ \mathrm{C}(8)-\mathrm{C}(9)-\mathrm{C}(10) & 119.8(6) \\ \mathrm{C}(8)-\mathrm{C}(9)-\mathrm{H}(9) & 120.1 \\ \mathrm{C}(10)-\mathrm{C}(9)-\mathrm{H}(9) & 120.1 \\ \mathrm{C}(5)-\mathrm{C}(10)-\mathrm{C}(9) & 121.2(5) \\ \mathrm{C}(5)-\mathrm{C}(10)-\mathrm{H}(10) & 119.4 \\ \mathrm{C}(9)-\mathrm{C}(10)-\mathrm{H}(10) & 119.4 \\ \mathrm{C}(16)-\mathrm{C}(11)-\mathrm{C}(12) & 118.7(5) \\ \mathrm{C}(16)-\mathrm{C}(11)-\mathrm{P}(1) & 119.7(4) \\ \mathrm{C}(12)-\mathrm{C}(11)-\mathrm{P}(1) & 121.6(4) \\ \mathrm{C}(13)-\mathrm{C}(12)-\mathrm{C}(11) & 120.8(6) \\ \mathrm{C}(13)-\mathrm{C}(12)-\mathrm{H}(12) & 119.6 \\ \mathrm{C}(11)-\mathrm{C}(12)-\mathrm{H}(12) & 119.6 \\ \mathrm{C}(14)-\mathrm{C}(13)-\mathrm{C}(12) & 119.4(6) \\ \mathrm{C}(14)-\mathrm{C}(13)-\mathrm{H}(13) & 120.3 \\ \mathrm{C}(12)-\mathrm{C}(13)-\mathrm{H}(13) & 120.3 \\ \mathrm{C}(13)-\mathrm{C}(14)-\mathrm{C}(15) & 121.4(6) \\ \mathrm{C}(13)-\mathrm{C}(14)-\mathrm{H}(14) & 119.3 \\ \mathrm{C}(15)-\mathrm{C}(14)-\mathrm{H}(14) & 119.3 \\ \mathrm{C}(14)-\mathrm{C}(15)-\mathrm{C}(16) & 119.0(6) \\ \mathrm{C}(14)-\mathrm{C}(15)-\mathrm{H}(15) & 120.5 \\ \mathrm{C}(16)-\mathrm{C}(15)-\mathrm{H}(15) & 120.5 \\ \mathrm{C}(11)-\mathrm{C}(16)-\mathrm{C}(15) & 120.7(5) \\ \mathrm{C}(11)-\mathrm{C}(16)-\mathrm{H}(16) & 119.7 \\ \mathrm{C}(15)-\mathrm{C}(16)-\mathrm{H}(16) & 119.7 \\ \mathrm{C}(18)-\mathrm{C}(17)-\mathrm{C}(22) & 118.2(5) \\ \mathrm{C}(18)-\mathrm{C}(17)-\mathrm{P}(1) & 117.6(4) \\ \mathrm{C}(22)-\mathrm{C}(17)-\mathrm{P}(1) & 124.1(4) \\ \mathrm{C}(17)-\mathrm{C}(18)-\mathrm{C}(19) & 121.7(6) \\ \mathrm{C}(17)-\mathrm{C}(18)-\mathrm{H}(18) & 119.2 \\ \mathrm{C}(19)-\mathrm{C}(18)-\mathrm{H}(18) & 119.2 \\ \mathrm{C}(20)-\mathrm{C}(19)-\mathrm{C}(18) & 118.5(7) \\ \mathrm{C}(10)-\mathrm{C}(19)-\mathrm{H}(19) & 120.8 \\ \mathrm{C}(21)-\mathrm{C}(19)-\mathrm{H}(19) & 120.8 \\ & 121.0(7) \\ & \end{array}$




\begin{tabular}{|c|c|}
\hline $\mathrm{C}(21)-\mathrm{C}(20)-\mathrm{H}(20)$ & 119.5 \\
\hline $\mathrm{C}(19)-\mathrm{C}(20)-\mathrm{H}(20)$ & 119.5 \\
\hline $\mathrm{C}(20)-\mathrm{C}(21)-\mathrm{C}(22)$ & $121.0(7)$ \\
\hline $\mathrm{C}(20)-\mathrm{C}(21)-\mathrm{H}(21)$ & 119.5 \\
\hline $\mathrm{C}(22)-\mathrm{C}(21)-\mathrm{H}(21)$ & 119.5 \\
\hline $\mathrm{C}(21)-\mathrm{C}(22)-\mathrm{C}(17)$ & $119.6(6)$ \\
\hline $\mathrm{C}(21)-\mathrm{C}(22)-\mathrm{H}(22)$ & 120.2 \\
\hline $\mathrm{C}(17)-\mathrm{C}(22)-\mathrm{H}(22)$ & 120.2 \\
\hline $\mathrm{C}(24)-\mathrm{C}(23)-\mathrm{C}(28)$ & $117.9(4)$ \\
\hline $\mathrm{C}(24)-\mathrm{C}(23)-\mathrm{P}(2)$ & $123.0(4)$ \\
\hline $\mathrm{C}(28)-\mathrm{C}(23)-\mathrm{P}(2)$ & $119.0(3)$ \\
\hline $\mathrm{C}(25)-\mathrm{C}(24)-\mathrm{C}(23)$ & $120.1(5)$ \\
\hline $\mathrm{C}(25)-\mathrm{C}(24)-\mathrm{H}(24)$ & 120.0 \\
\hline $\mathrm{C}(23)-\mathrm{C}(24)-\mathrm{H}(24)$ & 120.0 \\
\hline$C(26)-C(25)-C(24)$ & $121.0(5)$ \\
\hline $\mathrm{C}(26)-\mathrm{C}(25)-\mathrm{H}(25)$ & 119.5 \\
\hline $\mathrm{C}(24)-\mathrm{C}(25)-\mathrm{H}(25)$ & 119.5 \\
\hline $\mathrm{C}(27)-\mathrm{C}(26)-\mathrm{C}(25)$ & $119.6(5)$ \\
\hline $\mathrm{C}(27)-\mathrm{C}(26)-\mathrm{H}(26)$ & 120.2 \\
\hline $\mathrm{C}(25)-\mathrm{C}(26)-\mathrm{H}(26)$ & 120.2 \\
\hline $\mathrm{C}(26)-\mathrm{C}(27)-\mathrm{C}(28)$ & $119.8(5)$ \\
\hline $\mathrm{C}(26)-\mathrm{C}(27)-\mathrm{H}(27)$ & 120.1 \\
\hline $\mathrm{C}(28)-\mathrm{C}(27)-\mathrm{H}(27)$ & 120.1 \\
\hline $\mathrm{C}(27)-\mathrm{C}(28)-\mathrm{C}(23)$ & $121.6(5)$ \\
\hline $\mathrm{C}(27)-\mathrm{C}(28)-\mathrm{H}(28)$ & 119.2 \\
\hline $\mathrm{C}(23)-\mathrm{C}(28)-\mathrm{H}(28)$ & 119.2 \\
\hline$C(30)-C(29)-C(34)$ & $118.5(5)$ \\
\hline $\mathrm{C}(30)-\mathrm{C}(29)-\mathrm{P}(2)$ & $121.2(4)$ \\
\hline C(34)-C(29)-P(2) & $120.3(4)$ \\
\hline$C(29)-C(30)-C(31)$ & $120.7(5)$ \\
\hline $\mathrm{C}(29)-\mathrm{C}(30)-\mathrm{H}(30)$ & 119.6 \\
\hline $\mathrm{C}(31)-\mathrm{C}(30)-\mathrm{H}(30)$ & 119.6 \\
\hline$C(32)-C(31)-C(30)$ & $121.0(6)$ \\
\hline $\mathrm{C}(32)-\mathrm{C}(31)-\mathrm{H}(31)$ & 119.5 \\
\hline $\mathrm{C}(30)-\mathrm{C}(31)-\mathrm{H}(31)$ & 119.5 \\
\hline $\mathrm{C}(31)-\mathrm{C}(32)-\mathrm{C}(33)$ & $119.3(6)$ \\
\hline $\mathrm{C}(31)-\mathrm{C}(32)-\mathrm{H}(32)$ & 120.4 \\
\hline $\mathrm{C}(33)-\mathrm{C}(32)-\mathrm{H}(32)$ & 120.4 \\
\hline $\mathrm{C}(34)-\mathrm{C}(33)-\mathrm{C}(32)$ & $119.4(6)$ \\
\hline $\mathrm{C}(34)-\mathrm{C}(33)-\mathrm{H}(33)$ & 120.3 \\
\hline $\mathrm{C}(32)-\mathrm{C}(33)-\mathrm{H}(33)$ & 120.3 \\
\hline $\mathrm{C}(29)-\mathrm{C}(34)-\mathrm{C}(33)$ & $120.9(5)$ \\
\hline $\mathrm{C}(29)-\mathrm{C}(34)-\mathrm{H}(34)$ & 119.5 \\
\hline $\mathrm{C}(33)-\mathrm{C}(34)-\mathrm{H}(34)$ & 119.5 \\
\hline$C(36)-C(35)-C(40)$ & $118.4(4)$ \\
\hline $\mathrm{C}(36)-\mathrm{C}(35)-\mathrm{P}(2)$ & $119.7(4)$ \\
\hline
\end{tabular}




$\begin{array}{ll}\mathrm{C}(40)-\mathrm{C}(35)-\mathrm{P}(2) & 121.8(4) \\ \mathrm{C}(35)-\mathrm{C}(36)-\mathrm{C}(37) & 120.9(5) \\ \mathrm{C}(35)-\mathrm{C}(36)-\mathrm{H}(36) & 119.5 \\ \mathrm{C}(37)-\mathrm{C}(36)-\mathrm{H}(36) & 119.5 \\ \mathrm{C}(38)-\mathrm{C}(37)-\mathrm{C}(36) & 119.3(5) \\ \mathrm{C}(38)-\mathrm{C}(37)-\mathrm{H}(37) & 120.4 \\ \mathrm{C}(36)-\mathrm{C}(37)-\mathrm{H}(37) & 120.4 \\ \mathrm{C}(37)-\mathrm{C}(38)-\mathrm{C}(39) & 120.7(5) \\ \mathrm{C}(37)-\mathrm{C}(38)-\mathrm{H}(38) & 119.7 \\ \mathrm{C}(39)-\mathrm{C}(38)-\mathrm{H}(38) & 119.7 \\ \mathrm{C}(38)-\mathrm{C}(39)-\mathrm{C}(40) & 119.7(5) \\ \mathrm{C}(38)-\mathrm{C}(39)-\mathrm{H}(39) & 120.1 \\ \mathrm{C}(40)-\mathrm{C}(39)-\mathrm{H}(39) & 120.1 \\ \mathrm{C}(39)-\mathrm{C}(40)-\mathrm{C}(35) & 120.9(5) \\ \mathrm{C}(39)-\mathrm{C}(40)-\mathrm{H}(40) & 119.5 \\ \mathrm{C}(35)-\mathrm{C}(40)-\mathrm{H}(40) & 119.5 \\ \mathrm{C}(42)-\mathrm{C}(41)-\mathrm{N}(4) & 120.5(4) \\ \mathrm{C}(42)-\mathrm{C}(41)-\mathrm{Pd}(2) & 118.9(4) \\ \mathrm{N}(4)-\mathrm{C}(41)-\mathrm{Pd}(2) & 120.5(4) \\ \mathrm{C}(43)-\mathrm{C}(42)-\mathrm{C}(41) & 118.2(5) \\ \mathrm{C}(43)-\mathrm{C}(42)-\mathrm{H}(42) & 120.9 \\ \mathrm{C}(41)-\mathrm{C}(42)-\mathrm{H}(42) & 120.9 \\ \mathrm{~N}(3)-\mathrm{C}(43)-\mathrm{C}(42) & 126.1(5) \\ \mathrm{N}(3)-\mathrm{C}(43)-\mathrm{Cl}(5) & 113.9(4) \\ \mathrm{C}(42)-\mathrm{C}(43)-\mathrm{Cl}(5) & 120.0(5) \\ \mathrm{N}(3)-\mathrm{C}(44)-\mathrm{N}(4) & 127.1(5) \\ \mathrm{N}(3)-\mathrm{C}(44)-\mathrm{Cl}(6) & 116.3(4) \\ \mathrm{N}(4)-\mathrm{C}(44)-\mathrm{Cl}(6) & 116.6(4) \\ \mathrm{C}(50)-\mathrm{C}(45)-\mathrm{C}(46) & 119.1(5) \\ \mathrm{C}(50)-\mathrm{C}(45)-\mathrm{P}(3) & 123.8(5) \\ \mathrm{C}(46)-\mathrm{C}(45)-\mathrm{P}(3) & 117.0(4) \\ \mathrm{C}(45)-\mathrm{C}(46)-\mathrm{C}(47) & 119.9(6) \\ \mathrm{C}(45)-\mathrm{C}(46)-\mathrm{H}(46) & 120.1 \\ \mathrm{C}(47)-\mathrm{C}(46)-\mathrm{H}(46) & 120.1 \\ \mathrm{C}(48)-\mathrm{C}(47)-\mathrm{C}(46) & 119.2(7) \\ \mathrm{C}(48)-\mathrm{C}(47)-\mathrm{H}(47) & 120.4 \\ \mathrm{C}(46)-\mathrm{C}(47)-\mathrm{H}(47) & 120.4 \\ \mathrm{C}(49)-\mathrm{C}(48)-\mathrm{C}(47) & 121.5(7) \\ \mathrm{C}(49)-\mathrm{C}(48)-\mathrm{H}(48) & 119.3 \\ \mathrm{C}(47)-\mathrm{C}(48)-\mathrm{H}(48) & 119.3 \\ \mathrm{C}(48)-\mathrm{C}(49)-\mathrm{C}(50) & 120.6(7) \\ \mathrm{C}(48)-\mathrm{C}(49)-\mathrm{H}(49) & 119.7 \\ \mathrm{C}(50)-\mathrm{C}(49)-\mathrm{H}(49) & 119.7 \\ \mathrm{C}(45)-\mathrm{H}(50) & 119.7(6) \\ & 120.1 \\ \mathrm{C}(50)-\mathrm{H}(50) & 120.1 \\ & \end{array}$




$\begin{array}{ll}\mathrm{C}(56)-\mathrm{C}(51)-\mathrm{C}(52) & 118.8(5) \\ \mathrm{C}(56)-\mathrm{C}(51)-\mathrm{P}(3) & 120.7(4) \\ \mathrm{C}(52)-\mathrm{C}(51)-\mathrm{P}(3) & 120.5(4) \\ \mathrm{C}(53)-\mathrm{C}(52)-\mathrm{C}(51) & 120.9(6) \\ \mathrm{C}(53)-\mathrm{C}(52)-\mathrm{H}(52) & 119.6 \\ \mathrm{C}(51)-\mathrm{C}(52)-\mathrm{H}(52) & 119.6 \\ \mathrm{C}(52)-\mathrm{C}(53)-\mathrm{C}(54) & 119.8(6) \\ \mathrm{C}(52)-\mathrm{C}(53)-\mathrm{H}(53) & 120.1 \\ \mathrm{C}(54)-\mathrm{C}(53)-\mathrm{H}(53) & 120.1 \\ \mathrm{C}(53)-\mathrm{C}(54)-\mathrm{C}(55) & 119.9(6) \\ \mathrm{C}(53)-\mathrm{C}(54)-\mathrm{H}(54) & 120.1 \\ \mathrm{C}(55)-\mathrm{C}(54)-\mathrm{H}(54) & 120.1 \\ \mathrm{C}(54)-\mathrm{C}(55)-\mathrm{C}(56) & 119.9(6) \\ \mathrm{C}(54)-\mathrm{C}(55)-\mathrm{H}(55) & 120.1 \\ \mathrm{C}(56)-\mathrm{C}(55)-\mathrm{H}(55) & 120.1 \\ \mathrm{C}(51)-\mathrm{C}(56)-\mathrm{C}(55) & 120.7(6) \\ \mathrm{C}(51)-\mathrm{C}(56)-\mathrm{H}(56) & 119.6 \\ \mathrm{C}(55)-\mathrm{C}(56)-\mathrm{H}(56) & 119.6 \\ \mathrm{C}(58)-\mathrm{C}(57)-\mathrm{C}(62) & 117.2(5) \\ \mathrm{C}(58)-\mathrm{C}(57)-\mathrm{P}(3) & 123.0(4) \\ \mathrm{C}(62)-\mathrm{C}(57)-\mathrm{P}(3) & 119.8(4) \\ \mathrm{C}(57)-\mathrm{C}(58)-\mathrm{C}(59) & 121.8(6) \\ \mathrm{C}(57)-\mathrm{C}(58)-\mathrm{H}(58) & 119.1 \\ \mathrm{C}(59)-\mathrm{C}(58)-\mathrm{H}(58) & 119.1 \\ \mathrm{C}(60)-\mathrm{C}(59)-\mathrm{C}(58) & 119.7(7) \\ \mathrm{C}(60)-\mathrm{C}(59)-\mathrm{H}(59) & 120.2 \\ \mathrm{C}(58)-\mathrm{C}(59)-\mathrm{H}(59) & 120.2 \\ \mathrm{C}(59)-\mathrm{C}(60)-\mathrm{C}(61) & 119.6(7) \\ \mathrm{C}(59)-\mathrm{C}(60)-\mathrm{H}(60) & 120.2 \\ \mathrm{C}(61)-\mathrm{C}(60)-\mathrm{H}(60) & 120.2 \\ \mathrm{C}(60)-\mathrm{C}(61)-\mathrm{C}(62) & 119.7(7) \\ \mathrm{C}(60)-\mathrm{C}(61)-\mathrm{H}(61) & 120.1 \\ \mathrm{C}(62)-\mathrm{C}(61)-\mathrm{H}(61) & 120.1 \\ \mathrm{C}(57)-\mathrm{C}(62)-\mathrm{C}(61) & 121.8(6) \\ \mathrm{C}(57)-\mathrm{C}(62)-\mathrm{H}(62) & 119.1 \\ \mathrm{C}(61)-\mathrm{C}(62)-\mathrm{H}(62) & 119.1 \\ \mathrm{C}(64)-\mathrm{C}(63)-\mathrm{C}(68) & 118.3(5) \\ \mathrm{C}(64)-\mathrm{C}(63)-\mathrm{P}(4) & 123.9(4) \\ \mathrm{C}(68)-\mathrm{C}(63)-\mathrm{P}(4) & 117.9(4) \\ \mathrm{C}(63)-\mathrm{C}(64)-\mathrm{C}(65) & 119.9(5) \\ \mathrm{C}(63)-\mathrm{C}(64)-\mathrm{H}(64) & 120.0 \\ \mathrm{C}(65)-\mathrm{C}(64)-\mathrm{H}(64) & 120.0 \\ \mathrm{C}(66)-\mathrm{C}(65)-\mathrm{C}(64) & 121.7(6) \\ & 119.2 \\ \mathrm{C}(65) & 119.2 \\ \mathrm{C}(65)-\mathrm{C}(65) & 119.1(6) \\ \end{array}$




$\begin{array}{ll}\mathrm{C}(65)-\mathrm{C}(66)-\mathrm{H}(66) & 120.4 \\ \mathrm{C}(67)-\mathrm{C}(66)-\mathrm{H}(66) & 120.4 \\ \mathrm{C}(66)-\mathrm{C}(67)-\mathrm{C}(68) & 119.8(6) \\ \mathrm{C}(66)-\mathrm{C}(67)-\mathrm{H}(67) & 120.1 \\ \mathrm{C}(68)-\mathrm{C}(67)-\mathrm{H}(67) & 120.1 \\ \mathrm{C}(63)-\mathrm{C}(68)-\mathrm{C}(67) & 121.1(6) \\ \mathrm{C}(63)-\mathrm{C}(68)-\mathrm{H}(68) & 119.4 \\ \mathrm{C}(67)-\mathrm{C}(68)-\mathrm{H}(68) & 119.4 \\ \mathrm{C}(70)-\mathrm{C}(69)-\mathrm{C}(74) & 118.2(5) \\ \mathrm{C}(70)-\mathrm{C}(69)-\mathrm{P}(4) & 118.8(4) \\ \mathrm{C}(74)-\mathrm{C}(69)-\mathrm{P}(4) & 123.1(4) \\ \mathrm{C}(69)-\mathrm{C}(70)-\mathrm{C}(71) & 120.9(5) \\ \mathrm{C}(69)-\mathrm{C}(70)-\mathrm{H}(70) & 119.6 \\ \mathrm{C}(71)-\mathrm{C}(70)-\mathrm{H}(70) & 119.6 \\ \mathrm{C}(72)-\mathrm{C}(71)-\mathrm{C}(70) & 119.6(6) \\ \mathrm{C}(72)-\mathrm{C}(71)-\mathrm{H}(71) & 120.2 \\ \mathrm{C}(70)-\mathrm{C}(71)-\mathrm{H}(71) & 120.2 \\ \mathrm{C}(71)-\mathrm{C}(72)-\mathrm{C}(73) & 120.5(6) \\ \mathrm{C}(71)-\mathrm{C}(72)-\mathrm{H}(72) & 119.7 \\ \mathrm{C}(73)-\mathrm{C}(72)-\mathrm{H}(72) & 119.7 \\ \mathrm{C}(72)-\mathrm{C}(73)-\mathrm{C}(74) & 119.5(6) \\ \mathrm{C}(72)-\mathrm{C}(73)-\mathrm{H}(73) & 120.2 \\ \mathrm{C}(74)-\mathrm{C}(73)-\mathrm{H}(73) & 120.2 \\ \mathrm{C}(73)-\mathrm{C}(74)-\mathrm{C}(69) & 121.3(6) \\ \mathrm{C}(73)-\mathrm{C}(74)-\mathrm{H}(74) & 119.3 \\ \mathrm{C}(69)-\mathrm{C}(74)-\mathrm{H}(74) & 119.3 \\ \mathrm{C}(76)-\mathrm{C}(75)-\mathrm{C}(80) & 118.6(5) \\ \mathrm{C}(76)-\mathrm{C}(75)-\mathrm{P}(4) & 121.2(4) \\ \mathrm{C}(80)-\mathrm{C}(75)-\mathrm{P}(4) & 120.2(4) \\ \mathrm{C}(75)-\mathrm{C}(76)-\mathrm{C}(77) & 120.3(5) \\ \mathrm{C}(75)-\mathrm{C}(76)-\mathrm{H}(76) & 119.8 \\ \mathrm{C}(77)-\mathrm{C}(76)-\mathrm{H}(76) & 119.8 \\ \mathrm{C}(78)-\mathrm{C}(77)-\mathrm{C}(76) & 120.7(6) \\ \mathrm{C}(78)-\mathrm{C}(77)-\mathrm{H}(77) & 119.7 \\ \mathrm{C}(76)-\mathrm{C}(77)-\mathrm{H}(77) & 119.7 \\ \mathrm{C}(77)-\mathrm{C}(78)-\mathrm{C}(79) & 120.2(6) \\ \mathrm{C}(77)-\mathrm{C}(78)-\mathrm{H}(78) & 119.9 \\ \mathrm{C}(79)-\mathrm{C}(78)-\mathrm{H}(78) & 119.9 \\ \mathrm{C}(78)-\mathrm{C}(79)-\mathrm{C}(80) & 119.5(6) \\ \mathrm{C}(78)-\mathrm{C}(79)-\mathrm{H}(79) & 120.2 \\ \mathrm{C}(80)-\mathrm{C}(79)-\mathrm{H}(79) & 120.2 \\ \mathrm{C}(79)-\mathrm{C}(80)-\mathrm{C}(75) & 120.6(5) \\ \mathrm{C}(79)-\mathrm{C}(80)-\mathrm{H}(80) & 119.7 \\ \mathrm{C}(75)-\mathrm{C}(80)-\mathrm{H}(80) & 119.7 \\ \mathrm{C}(1) & 112.9(4) \\ & \\ \mathrm{C}(4) .9(4)\end{array}$




$\begin{array}{lc}\mathrm{C}(44)-\mathrm{N}(3)-\mathrm{C}(43) & 112.9(4) \\ \mathrm{C}(41)-\mathrm{N}(4)-\mathrm{C}(44) & 115.1(4) \\ \mathrm{C}(11)-\mathrm{P}(1)-\mathrm{C}(5) & 103.1(2) \\ \mathrm{C}(11)-\mathrm{P}(1)-\mathrm{C}(17) & 108.9(2) \\ \mathrm{C}(5)-\mathrm{P}(1)-\mathrm{C}(17) & 105.7(2) \\ \mathrm{C}(11)-\mathrm{P}(1)-\mathrm{Pd}(1) & 112.35(16) \\ \mathrm{C}(5)-\mathrm{P}(1)-\mathrm{Pd}(1) & 118.68(15) \\ \mathrm{C}(17)-\mathrm{P}(1)-\mathrm{Pd}(1) & 107.66(16) \\ \mathrm{C}(23)-\mathrm{P}(2)-\mathrm{C}(29) & 104.0(2) \\ \mathrm{C}(23)-\mathrm{P}(2)-\mathrm{C}(35) & 103.2(2) \\ \mathrm{C}(29)-\mathrm{P}(2)-\mathrm{C}(35) & 106.4(2) \\ \mathrm{C}(23)-\mathrm{P}(2)-\mathrm{Pd}(1) & 116.38(14) \\ \mathrm{C}(29)-\mathrm{P}(2)-\mathrm{Pd}(1) & 110.47(15) \\ \mathrm{C}(35)-\mathrm{P}(2)-\mathrm{Pd}(1) & 115.25(15) \\ \mathrm{C}(57)-\mathrm{P}(3)-\mathrm{C}(45) & 105.9(3) \\ \mathrm{C}(57)-\mathrm{P}(3)-\mathrm{C}(51) & 104.4(2) \\ \mathrm{C}(45)-\mathrm{P}(3)-\mathrm{C}(51) & 102.5(2) \\ \mathrm{C}(57)-\mathrm{P}(3)-\mathrm{Pd}(2) & 106.58(17) \\ \mathrm{C}(45)-\mathrm{P}(3)-\mathrm{Pd}(2) & 114.35(16) \\ \mathrm{C}(51)-\mathrm{P}(3)-\mathrm{Pd}(2) & 121.81(17) \\ \mathrm{C}(63)-\mathrm{P}(4)-\mathrm{C}(75) & 107.1(2) \\ \mathrm{C}(63)-\mathrm{P}(4)-\mathrm{C}(69) & 104.3(2) \\ \mathrm{C}(75)-\mathrm{P}(4)-\mathrm{C}(69) & 101.5(2) \\ \mathrm{C}(63)-\mathrm{P}(4)-\mathrm{Pd}(2) & 109.83(15) \\ \mathrm{C}(75)-\mathrm{P}(4)-\mathrm{Pd}(2) & 116.25(16) \\ \mathrm{C}(69)-\mathrm{P}(4)-\mathrm{Pd}(2) & 116.75(15) \\ \mathrm{C}(1)-\mathrm{Pd}(1)-\mathrm{P}(1) & 89.88(12) \\ \mathrm{C}(1)-\mathrm{Pd}(1)-\mathrm{P}(2) & 91.01(12) \\ \mathrm{P}(1)-\mathrm{Pd}(1)-\mathrm{P}(2) & 177.53(5) \\ \mathrm{C}(1)-\mathrm{Pd}(1)-\mathrm{Cl}(1) & 177.08(13) \\ \mathrm{P}(1)-\mathrm{Pd}(1)-\mathrm{Cl}(1) & 87.67(4) \\ \mathrm{P}(2)-\mathrm{Pd}(1)-\mathrm{Cl}(1) & 91.37(4) \\ \mathrm{C}(41)-\mathrm{Pd}(2)-\mathrm{P}(4) & 88.39(12) \\ \mathrm{C}(41)-\mathrm{Pd}(2)-\mathrm{P}(3) & 89.68(13) \\ \mathrm{P}(4)-\mathrm{Pd}(2)-\mathrm{P}(3) & 173.21(5) \\ \mathrm{C}(41)-\mathrm{Pd}(2)-\mathrm{Cl}(4) & 174.63(14) \\ \mathrm{P}(4)-\mathrm{Pd}(2)-\mathrm{Cl}(4) & 88.75(4) \\ \mathrm{P}(3)-\mathrm{Pd}(2)-\mathrm{Cl}(4) & 93.68(5) \\ & \end{array}$

Symmetry transformations used to generate equivalent atoms: 
Table 4. Anisotropic displacement parameters $\left(\AA^{2} \times 10^{3}\right)$ for compound 6. The anisotropic displacement factor exponent takes the form: $-2 \pi^{2}\left[h^{2} a^{* 2} U^{11}+\ldots+2 h k a^{*} b^{*} U^{12}\right]$

\begin{tabular}{|c|c|c|c|c|c|c|}
\hline & $\mathrm{U}^{11}$ & $\mathrm{U}^{22}$ & $\mathrm{U}^{33}$ & $\mathrm{U}^{23}$ & $\mathrm{U}^{13}$ & $\mathrm{U}^{12}$ \\
\hline $\mathrm{C}(1)$ & $45(3)$ & $36(3)$ & $39(3)$ & $0(2)$ & $15(2)$ & $-3(2)$ \\
\hline $\mathrm{C}(2)$ & $42(3)$ & $38(3)$ & $33(3)$ & $-9(2)$ & $1(2)$ & $10(2)$ \\
\hline $\mathrm{C}(3)$ & $53(3)$ & $52(3)$ & $51(3)$ & $-2(3)$ & $0(3)$ & $14(3)$ \\
\hline$C(4)$ & $65(4)$ & $39(3)$ & $44(3)$ & $0(2)$ & $9(3)$ & $-3(3)$ \\
\hline$C(5)$ & $36(3)$ & $44(3)$ & $49(3)$ & $4(2)$ & $12(2)$ & $-3(2)$ \\
\hline$C(6)$ & $56(4)$ & $112(5)$ & $58(4)$ & $-5(4)$ & $14(3)$ & $9(4)$ \\
\hline $\mathrm{C}(7)$ & $60(4)$ & $183(8)$ & $75(5)$ & $-22(5)$ & $16(4)$ & $27(5)$ \\
\hline $\mathrm{C}(8)$ & $67(5)$ & 121(6) & $83(5)$ & $-7(4)$ & $37(4)$ & $14(4)$ \\
\hline C(9) & $75(5)$ & $107(5)$ & $66(4)$ & $-32(4)$ & $30(4)$ & $-11(4)$ \\
\hline$C(10)$ & $44(3)$ & $78(4)$ & $61(4)$ & $-7(3)$ & $16(3)$ & $-3(3)$ \\
\hline $\mathrm{C}(11)$ & $55(3)$ & $33(3)$ & $47(3)$ & $-7(2)$ & $13(2)$ & $-4(2)$ \\
\hline$C(12)$ & $69(4)$ & $56(4)$ & $79(4)$ & $19(3)$ & $22(3)$ & $-1(3)$ \\
\hline$C(13)$ & $117(6)$ & $74(5)$ & $82(5)$ & $35(4)$ & $26(5)$ & $17(5)$ \\
\hline $\mathrm{C}(14)$ & $123(6)$ & $60(4)$ & $58(4)$ & $12(3)$ & $-5(4)$ & $24(4)$ \\
\hline$C(15)$ & $71(4)$ & $66(4)$ & $69(4)$ & $-7(3)$ & $-8(3)$ & $11(3)$ \\
\hline$C(16)$ & $61(4)$ & $48(3)$ & $52(3)$ & $-2(3)$ & $7(3)$ & $-2(3)$ \\
\hline$C(17)$ & $42(3)$ & $52(3)$ & $47(3)$ & $-2(3)$ & $12(2)$ & $-5(2)$ \\
\hline $\mathrm{C}(18)$ & $75(4)$ & $72(4)$ & $67(4)$ & $3(3)$ & $-1(3)$ & $-28(3)$ \\
\hline C(19) & $84(5)$ & $133(7)$ & $59(4)$ & $6(5)$ & $-1(4)$ & $-26(5)$ \\
\hline$C(20)$ & $91(6)$ & $146(8)$ & $77(6)$ & $-33(6)$ & $4(4)$ & $-35(6)$ \\
\hline $\mathrm{C}(21)$ & $94(5)$ & $73(5)$ & $114(6)$ & $-34(5)$ & $8(5)$ & $-24(4)$ \\
\hline$C(22)$ & $67(4)$ & $58(4)$ & $82(4)$ & $-6(3)$ & $10(3)$ & $-3(3)$ \\
\hline$C(23)$ & $39(3)$ & $33(3)$ & $43(3)$ & $1(2)$ & $12(2)$ & $-6(2)$ \\
\hline $\mathrm{C}(24)$ & $66(4)$ & $49(3)$ & $48(3)$ & $4(3)$ & $4(3)$ & $10(3)$ \\
\hline$C(25)$ & $89(4)$ & $43(3)$ & $85(5)$ & $18(3)$ & $20(4)$ & $25(3)$ \\
\hline$C(26)$ & $88(5)$ & $36(3)$ & $89(5)$ & $-10(3)$ & $28(4)$ & $2(3)$ \\
\hline$C(27)$ & $78(4)$ & $39(3)$ & $65(4)$ & $-6(3)$ & $13(3)$ & $-4(3)$ \\
\hline $\mathrm{C}(28)$ & $66(3)$ & $33(3)$ & $54(3)$ & $-2(2)$ & $8(3)$ & $0(2)$ \\
\hline$C(29)$ & $39(3)$ & $35(3)$ & $46(3)$ & $-5(2)$ & $8(2)$ & $0(2)$ \\
\hline$C(30)$ & $49(3)$ & $103(5)$ & $44(3)$ & $-2(3)$ & $13(3)$ & $-4(3)$ \\
\hline $\mathrm{C}(31)$ & $72(4)$ & $135(6)$ & $44(4)$ & $4(4)$ & $22(3)$ & $21(4)$ \\
\hline $\mathrm{C}(32)$ & $93(6)$ & $105(6)$ & $52(4)$ & $-19(4)$ & $-3(4)$ & $26(4)$ \\
\hline$C(33)$ & $68(4)$ & $87(5)$ & $83(5)$ & $-6(4)$ & $-21(4)$ & $-8(4)$ \\
\hline $\mathrm{C}(34)$ & $59(4)$ & $60(4)$ & $60(4)$ & $-3(3)$ & $3(3)$ & $0(3)$ \\
\hline$C(35)$ & $40(3)$ & $45(3)$ & $38(3)$ & $-2(2)$ & $14(2)$ & $-1(2)$ \\
\hline$C(36)$ & $49(3)$ & $47(3)$ & $61(3)$ & $3(3)$ & $6(3)$ & $5(3)$ \\
\hline C(37) & $59(4)$ & $59(4)$ & $85(4)$ & $-3(3)$ & $3(3)$ & $17(3)$ \\
\hline $\mathrm{C}(38)$ & $42(3)$ & $83(5)$ & $89(5)$ & $-9(4)$ & $-4(3)$ & $5(3)$ \\
\hline C(39) & $44(4)$ & $77(4)$ & $89(4)$ & $-5(4)$ & $7(3)$ & $-22(3)$ \\
\hline $\mathrm{C}(40)$ & $47(3)$ & $62(4)$ & $63(3)$ & $8(3)$ & $6(3)$ & $-3(3)$ \\
\hline $\mathrm{C}(41)$ & $34(3)$ & $36(3)$ & $53(3)$ & $-4(2)$ & $12(2)$ & $2(2)$ \\
\hline
\end{tabular}




\begin{tabular}{|c|c|c|c|c|c|c|}
\hline $\mathrm{C}(42)$ & $46(3)$ & $39(3)$ & $39(3)$ & $-10(2)$ & $6(2)$ & $-8(2)$ \\
\hline$C(43)$ & $74(4)$ & $64(4)$ & $47(3)$ & $5(3)$ & $15(3)$ & $7(3)$ \\
\hline $\mathrm{C}(44)$ & $46(3)$ & $43(3)$ & $77(4)$ & $2(3)$ & $9(3)$ & $-4(3)$ \\
\hline$C(45)$ & $52(3)$ & $47(3)$ & $69(4)$ & $-13(3)$ & $26(3)$ & $0(3)$ \\
\hline$C(46)$ & $64(4)$ & $56(4)$ & $87(5)$ & $2(3)$ & $23(3)$ & $12(3)$ \\
\hline $\mathrm{C}(47)$ & $98(5)$ & $66(5)$ & $123(6)$ & $22(5)$ & $43(5)$ & $19(4)$ \\
\hline $\mathrm{C}(48)$ & $129(7)$ & $48(4)$ & $151(8)$ & $4(5)$ & $71(6)$ & $-2(4)$ \\
\hline$C(49)$ & $126(7)$ & $55(5)$ & $131(7)$ & $-25(5)$ & $49(6)$ & $-21(4)$ \\
\hline $\mathrm{C}(50)$ & $99(5)$ & $60(4)$ & $86(5)$ & $-19(4)$ & $34(4)$ & $-11(4)$ \\
\hline $\mathrm{C}(51)$ & $47(3)$ & $59(3)$ & $42(3)$ & $-4(3)$ & $12(2)$ & $5(3)$ \\
\hline$C(52)$ & $56(4)$ & $76(4)$ & $89(4)$ & $-8(3)$ & $25(3)$ & $-3(3)$ \\
\hline $\mathrm{C}(53)$ & $65(4)$ & $93(5)$ & $98(5)$ & $-6(4)$ & $30(4)$ & $15(4)$ \\
\hline $\mathrm{C}(54)$ & $44(4)$ & $114(6)$ & $96(5)$ & $-5(4)$ & $12(3)$ & $-1(4)$ \\
\hline$C(55)$ & $58(4)$ & $94(5)$ & $89(5)$ & $-5(4)$ & $6(3)$ & $-11(4)$ \\
\hline$C(56)$ & $49(3)$ & $78(4)$ & $62(4)$ & $-7(3)$ & $3(3)$ & $7(3)$ \\
\hline$C(57)$ & $50(3)$ & $72(4)$ & $51(3)$ & $-8(3)$ & $15(3)$ & $4(3)$ \\
\hline $\mathrm{C}(58)$ & $61(4)$ & 228(9) & $51(4)$ & $-3(5)$ & $13(3)$ & $0(5)$ \\
\hline C(59) & $70(5)$ & $379(16)$ & $59(5)$ & $0(7)$ & $15(4)$ & $-14(8)$ \\
\hline$C(60)$ & $118(7)$ & $281(13)$ & $44(4)$ & $12(6)$ & $0(5)$ & $20(8)$ \\
\hline $\mathrm{C}(61)$ & $90(6)$ & $317(14)$ & $71(5)$ & $-14(7)$ & $-15(5)$ & $82(7)$ \\
\hline$C(62)$ & $75(5)$ & $235(10)$ & $58(4)$ & $-25(5)$ & $8(4)$ & $53(6)$ \\
\hline $\mathrm{C}(63)$ & $45(3)$ & $48(3)$ & $51(3)$ & $0(2)$ & $21(2)$ & $1(2)$ \\
\hline$C(64)$ & $58(4)$ & $52(4)$ & $67(4)$ & $1(3)$ & $2(3)$ & $0(3)$ \\
\hline$C(65)$ & $80(5)$ & $56(4)$ & $84(4)$ & $-17(4)$ & $11(4)$ & $-3(4)$ \\
\hline$C(66)$ & $68(4)$ & $90(5)$ & $59(4)$ & $-25(4)$ & $14(3)$ & $-18(4)$ \\
\hline $\mathrm{C}(67)$ & $68(4)$ & $94(5)$ & $73(4)$ & $-2(4)$ & $-13(3)$ & $-2(4)$ \\
\hline $\mathrm{C}(68)$ & $67(4)$ & $65(4)$ & $82(4)$ & $-2(4)$ & $-12(3)$ & $10(3)$ \\
\hline C(69) & $38(3)$ & $44(3)$ & $52(3)$ & $9(2)$ & $15(2)$ & $4(2)$ \\
\hline $\mathrm{C}(70)$ & $50(3)$ & $57(3)$ & $67(4)$ & $10(3)$ & $16(3)$ & $3(3)$ \\
\hline $\mathrm{C}(71)$ & $42(3)$ & $69(4)$ & $106(5)$ & $20(4)$ & $10(3)$ & $-6(3)$ \\
\hline $\mathrm{C}(72)$ & $70(5)$ & $108(6)$ & $105(6)$ & $46(5)$ & $35(4)$ & $-3(4)$ \\
\hline$C(73)$ & $94(6)$ & $152(7)$ & $68(5)$ & $24(5)$ & $30(4)$ & $-18(5)$ \\
\hline $\mathrm{C}(74)$ & $68(4)$ & $93(5)$ & $62(4)$ & $5(3)$ & $33(3)$ & $-19(3)$ \\
\hline $\mathrm{C}(75)$ & $51(3)$ & $36(3)$ & $52(3)$ & $-4(2)$ & $14(3)$ & $-6(2)$ \\
\hline $\mathrm{C}(76)$ & $61(4)$ & $78(4)$ & $53(3)$ & $7(3)$ & $8(3)$ & $3(3)$ \\
\hline $\mathrm{C}(77)$ & $87(5)$ & $92(5)$ & $59(4)$ & $13(4)$ & $2(4)$ & $5(4)$ \\
\hline $\mathrm{C}(78)$ & $69(5)$ & $73(4)$ & $83(5)$ & $18(4)$ & $-25(4)$ & $-3(4)$ \\
\hline C(79) & $50(4)$ & $61(4)$ & $107(5)$ & $13(4)$ & $13(4)$ & $1(3)$ \\
\hline $\mathrm{C}(80)$ & $55(3)$ & $57(3)$ & $63(4)$ & $18(3)$ & $10(3)$ & $7(3)$ \\
\hline $\mathrm{Cl}(1)$ & $64(1)$ & $35(1)$ & $70(1)$ & $-9(1)$ & $31(1)$ & $4(1)$ \\
\hline $\mathrm{Cl}(2)$ & $90(1)$ & $126(2)$ & $102(1)$ & $-33(1)$ & $-20(1)$ & $64(1)$ \\
\hline $\mathrm{Cl}(3)$ & 119(1) & $82(1)$ & $62(1)$ & $-15(1)$ & $-18(1)$ & $-15(1)$ \\
\hline $\mathrm{Cl}(4)$ & $72(1)$ & $44(1)$ & 117(1) & $14(1)$ & $51(1)$ & $-3(1)$ \\
\hline $\mathrm{Cl}(5)$ & $158(2)$ & $122(2)$ & 61(1) & $18(1)$ & $13(1)$ & 1(1) \\
\hline $\mathrm{Cl}(6)$ & $94(1)$ & $79(1)$ & $133(2)$ & $-2(1)$ & $-13(1)$ & $-40(1)$ \\
\hline $\mathrm{N}(1)$ & $66(3)$ & $51(3)$ & $57(3)$ & $-8(2)$ & $9(2)$ & $10(2)$ \\
\hline
\end{tabular}




$\begin{array}{lcccccc}\mathrm{N}(2) & 51(3) & 43(2) & 56(3) & 3(2) & 11(2) & -4(2) \\ \mathrm{N}(3) & 68(3) & 59(3) & 71(3) & 13(3) & 17(3) & -4(3) \\ \mathrm{N}(4) & 54(3) & 48(3) & 72(3) & 1(2) & 14(2) & -3(2) \\ \mathrm{P}(1) & 40(1) & 36(1) & 44(1) & 3(1) & 13(1) & -3(1) \\ \mathrm{P}(2) & 37(1) & 32(1) & 41(1) & 2(1) & 13(1) & 0(1) \\ \mathrm{P}(3) & 48(1) & 45(1) & 49(1) & -5(1) & 19(1) & 1(1) \\ \mathrm{P}(4) & 41(1) & 39(1) & 46(1) & 2(1) & 16(1) & 0(1) \\ \mathrm{Pd}(1) & 40(1) & 28(1) & 45(1) & 0(1) & 17(1) & 1(1) \\ \operatorname{Pd}(2) & 45(1) & 36(1) & 48(1) & -1(1) & 20(1) & -3(1)\end{array}$

Table 5. Hydrogen coordinates ( $\left.\times 10^{4}\right)$ and isotropic displacement parameters $\left(\AA^{2} \times 10^{3}\right)$ for compound 6.

\begin{tabular}{lrrrr}
\hline & $\mathrm{x}$ & $\mathrm{y}$ & $\mathrm{z}$ & $\mathrm{U}(\mathrm{eq})$ \\
\hline $\mathrm{H}(2)$ & 1892 & 6909 & 1588 & 46 \\
$\mathrm{H}(6)$ & 2602 & 9470 & 1197 & 90 \\
$\mathrm{H}(7)$ & 3149 & 8537 & 710 & 127 \\
$\mathrm{H}(8)$ & 2824 & 7670 & -84 & 105 \\
$\mathrm{H}(9)$ & 1962 & 7847 & -437 & 96 \\
$\mathrm{H}(10)$ & 1407 & 8779 & 43 & 72 \\
$\mathrm{H}(12)$ & 1672 & 11545 & 279 & 80 \\
$\mathrm{H}(13)$ & 1163 & 12770 & -314 & 108 \\
$\mathrm{H}(14)$ & 278 & 12709 & -389 & 99 \\
$\mathrm{H}(15)$ & -110 & 11500 & 145 & 85 \\
$\mathrm{H}(16)$ & 405 & 10331 & 772 & 65 \\
$\mathrm{H}(18)$ & 2066 & 9319 & 2058 & 88 \\
$\mathrm{H}(19)$ & 2539 & 10329 & 2769 & 112 \\
$\mathrm{H}(20)$ & 2573 & 12342 & 2733 & 127 \\
$\mathrm{H}(21)$ & 2227 & 13315 & 1982 & 114 \\
$\mathrm{H}(22)$ & 1782 & 12322 & 1256 & 83 \\
$\mathrm{H}(24)$ & 1022 & 5282 & 2500 & 66 \\
$\mathrm{H}(25)$ & 1141 & 3340 & 2302 & 86 \\
$\mathrm{H}(26)$ & 788 & 2583 & 1483 & 83 \\
$\mathrm{H}(27)$ & 305 & 3775 & 855 & 72 \\
$\mathrm{H}(28)$ & 167 & 5703 & 1052 & 61 \\
$\mathrm{H}(30)$ & 65 & 6636 & 2827 & 77 \\
$\mathrm{H}(31)$ & 328 & 6752 & 3730 & 99 \\
$\mathrm{H}(32)$ & 1074 & 7719 & 4085 & 103 \\
$\mathrm{H}(33)$ & 1600 & 8486 & 3523 & 100 \\
$\mathrm{H}(34)$ & 1348 & 8307 & 2613 & 72 \\
$\mathrm{H}(36)$ & -186 & 9147 & 1507 & 63 \\
$\mathrm{H}(37)$ & -1078 & 9455 & 1331 & 82 \\
$\mathrm{H}(38)$ & -1630 & 7951 & 1461 & 88 \\
$\mathrm{H}(39)$ & -1303 & 6159 & 1774 & 85 \\
& & & &
\end{tabular}




\begin{tabular}{|c|c|c|c|c|}
\hline $\mathrm{H}(40)$ & -419 & 5858 & 1955 & 69 \\
\hline $\mathrm{H}(42)$ & 4154 & 1391 & 4734 & 50 \\
\hline $\mathrm{H}(46)$ & 4902 & -131 & 4143 & 81 \\
\hline $\mathrm{H}(47)$ & 4831 & -2078 & 4408 & 111 \\
\hline $\mathrm{H}(48)$ & 4400 & -3405 & 3819 & 124 \\
\hline $\mathrm{H}(49)$ & 4070 & -2884 & 2973 & 121 \\
\hline $\mathrm{H}(50)$ & 4135 & -962 & 2690 & 95 \\
\hline $\mathrm{H}(52)$ & 5437 & -253 & 3127 & 86 \\
\hline $\mathrm{H}(53)$ & 6305 & 46 & 3117 & 100 \\
\hline $\mathrm{H}(54)$ & 6663 & 1888 & 3298 & 101 \\
\hline $\mathrm{H}(55)$ & 6135 & 3458 & 3419 & 97 \\
\hline $\mathrm{H}(56)$ & 5259 & 3156 & 3415 & 76 \\
\hline $\mathrm{H}(58)$ & 4929 & 978 & 2234 & 136 \\
\hline $\mathrm{H}(59)$ & 4567 & 1082 & 1350 & 202 \\
\hline $\mathrm{H}(60)$ & 3719 & 1633 & 1105 & 179 \\
\hline $\mathrm{H}(61)$ & 3206 & 1774 & 1738 & 195 \\
\hline $\mathrm{H}(62)$ & 3576 & 1706 & 2619 & 148 \\
\hline $\mathrm{H}(64)$ & 3435 & 6082 & 4320 & 72 \\
\hline $\mathrm{H}(65)$ & 3906 & 7243 & 4977 & 89 \\
\hline $\mathrm{H}(66)$ & 4564 & 6493 & 5575 & 86 \\
\hline $\mathrm{H}(67)$ & 4770 & 4527 & 5533 & 98 \\
\hline $\mathrm{H}(68)$ & 4321 & 3367 & 4869 & 89 \\
\hline $\mathrm{H}(70)$ & 2652 & 1966 & 3764 & 68 \\
\hline $\mathrm{H}(71)$ & 2116 & 861 & 4204 & 88 \\
\hline $\mathrm{H}(72)$ & 2162 & 1083 & 5104 & 110 \\
\hline $\mathrm{H}(73)$ & 2736 & 2403 & 5570 & 123 \\
\hline $\mathrm{H}(74)$ & 3268 & 3494 & 5133 & 86 \\
\hline $\mathrm{H}(76)$ & 3403 & 4445 & 2969 & 77 \\
\hline $\mathrm{H}(77)$ & 2814 & 5540 & 2389 & 97 \\
\hline $\mathrm{H}(78)$ & 2076 & 6243 & 2641 & 96 \\
\hline $\mathrm{H}(79)$ & 1899 & 5810 & 3473 & 87 \\
\hline $\mathrm{H}(80)$ & 2497 & 4754 & 4069 & 70 \\
\hline
\end{tabular}

